\title{
Strong homotopy Lie algebras, homotopy Poisson manifolds and Courant algebroids*
}

\author{
Honglei Lang ${ }^{a}$, Yunhe Sheng ${ }^{b}$ and Xiaomeng $\mathrm{Xu}^{c}$ \\ ${ }^{a}$ Department of Mathematics, Peking University, Beijing 100871, China \\ ${ }^{b}$ Department of Mathematics, Jilin University, Changchun 130012, China \\ \& Kavli Institute for Theoretical Physics China, CAS, Beijing 100190, China \\ ${ }^{c}$ Section of Mathematics, University of Geneva \\ 2-4 Rue de Lièvre, c.p. 64, 1211-Genève 4, Switzerland. \\ email: hllang@pku.edu.cn, shengyh@jlu.edu.cn, Xiaomeng.Xu@unige.ch
}

\begin{abstract}
We study Maurer-Cartan elements on homotopy Poisson manifolds of degree $n$. They unify many twisted or homotopy structures in Poisson geometry and mathematical physics, such as twisted Poisson manifolds, quasi-Poisson $\mathfrak{g}$-manifolds, and twisted Courant algebroids. Using the fact that the dual of an $n$-term $L_{\infty}$-algebra is a homotopy Poisson manifold of degree $n-1$, we obtain a Courant algebroid from a 2 -term $L_{\infty}$-algebra $\mathfrak{g}$ via the degree 2 symplectic NQ-manifold $T^{*}[2] \mathfrak{g}^{*}[1]$. By integrating the Lie quasi-bialgebroid associated to the Courant algebroid, we obtain a Lie-quasiPoisson groupoid from a 2 -term $L_{\infty}$-algebra, which is proposed to be the geometric structure on the dual of a Lie 2-algebra. These results lead to a construction of a new 2-term $L_{\infty}$-algebra from a given one, which could produce many interesting examples.
\end{abstract}

\section{Introduction}

The concept of an $L_{\infty}$-algebra (sometimes called a strongly homotopy (sh) Lie algebra) was originally introduced in [22, 38, as a model for "Lie algebras that satisfy the Jacobi identity up to all higher homotopies". A Lie 2-algebra is a categorification of a Lie algebra. It is well-known that the category of Lie 2-algebras is equivalent to the category of 2 -term $L_{\infty}$-algebras 4]. The structure of a Lie 2 -algebra or a 2 -term $L_{\infty}$-algebra appears in many areas such as string theory, higher symplectic geometry [5, 6], and Courant algebroids [27.

A homotopy Poisson algebra is a graded commutative algebra with an $L_{\infty}$-structure whose brackets satisfy the Leibniz rule. It has appeared in Voronov's work [17, 40] under the name "higher Poisson structures" and Cattaneo and Felder's work [10] under the name " $P_{\infty}$-structures". See also [8, 26] for more recent work involving this structure. A homotopy Poisson algebra structure of degree $n$ on a graded commutative algebra $\mathfrak{a}$ means that there is a homotopy Poisson algebra structure on the shifted space

\footnotetext{
${ }^{0}$ Keyword: $L_{\infty}$-algebras, Lie 2-algebras, homotopy Poisson manifolds, Courant algebroids, symplectic NQ-manifolds, Maurer-Cartan elements

${ }^{0} M S C: 53 \mathrm{D} 17$, 17B99.

* Research supported by NSFC (11101179,11471139) and NSF of Jilin Province (20140520054JH). Xiaomeng Xu was partially supported by the SNSF grants P2GEP2-165118 and NCCR SwissMAP.
} 
$\mathfrak{a}[n]$. One interesting example of homotopy Poisson manifolds is given by the dual $\mathfrak{g}^{*}[n-1]$ of an $n$-term $L_{\infty}$-algebra $\mathfrak{g}=\mathfrak{g}_{0} \oplus \mathfrak{g}_{-1} \oplus \cdots \oplus \mathfrak{g}_{1-n}$, which turns out to be a homotopy Poisson manifold of degree $n-1$. This generalizes the fact that there is a Lie-Poisson structure on the dual space of a Lie algebra to the case of $n$-term $L_{\infty}$-algebras.

It is known that there is a one-to-one correspondence between Poisson manifolds and symplectic NQ-manifolds of degree 1 ,

$$
(M, \pi) \leftrightarrow\left(T^{*}[1] M, Q=\{\pi, \cdot\}\right),
$$

where the bracket $\{\cdot, \cdot\}$ is the canonical Poisson bracket on the cotangent bundle of the manifold $M$. One can further study the cotangent bundle of a homotopy Poisson manifold $\mathcal{M}$ of degree $n$. The shifted cotangent bundle $T^{*}[n+1] \mathcal{M}$ is a symplectic NQ-manifold of degree $n+1$. Then we study Maurer-Cartan elements on a degree $n$ homotopy Poisson manifold $\mathcal{M}$, which are solutions of the Maurer-Cartan equation associated to the $L_{\infty}$-structure on the function $\operatorname{ring} C^{\infty}(\mathcal{M})$. Recall that there are many types of twisted or homotopy structures in Poisson geometry and mathematical physics. See 2, 14, 19, 20, 29, 33, 34 , for lists of such examples. We provide a unified framework to describe these structures in which they are viewed as solutions of the Maurer-Cartan equation. For example, given a manifold $M$, the cotangent bundle $T^{*}[1] M$, equipped with the Schouten bracket $l_{2}=[\cdot, \cdot]_{S}$, is a homotopy Poisson manifold of degree 1. Furthermore, a degree 2 function $\pi \in C^{\infty}\left(T^{*}[1] M\right)$ is a Maurer-Cartan element on $T^{*}[1] M$ if and only if $\pi$ is a Poisson tensor on $M$; in the twisted case, given a closed 3-form $H$, the cotangent bundle $T^{*}[1] M$ with $l_{2}=[\cdot, \cdot]_{S}$ and a ternary bracket $l_{3}=H(\cdot, \cdot, \cdot)$ on $C^{\infty}\left(T^{*}[1] M\right)$ is a new homotopy Poisson manifold of degree 1, and a degree 2 function $\pi$ satisfies the Maurer-Cartan equation if and only if $\pi$ is a twisted Poisson tensor.

A homotopy Poisson manifold $\mathcal{M}$ of degree $n$ is called symplectic if its binary bracket is non-degenerate (See [17 for the $\mathbb{Z}_{2}$-graded case). If $\mathcal{M}$ is a symplectic $\mathrm{N}$-manifold of degree $n$, then a function $\alpha$ of degree $n+1$, satisfying the Maurer-Cartan equation $l_{2}(\alpha, \alpha)=0$, induces a differential $Q=l_{2}(\alpha, \cdot)$ of degree 1 on $\mathcal{M}$. This shows that a differential graded symplectic manifold or symplectic NQ-manifold is a special homotopy symplectic manifold with a Maurer-Cartan element. A general degree $n$ homotopy symplectic manifold $\left(\mathcal{M},\left\{l_{i}\right\}_{1 \leq i<\infty}\right)$ with $l_{1}=0$ is therefore a homotopy version of a symplectic $\mathrm{N}$-manifold. In this case, the Maurer-Cartan equation becomes

$$
\frac{1}{2 !} l_{2}(\alpha, \alpha)-\frac{1}{3 !} l_{3}(\alpha, \alpha, \alpha)+\cdots=0,
$$

which can be viewed as a homotopy version of the classical master equation. In order to relate this to physical applications, we prove that homotopy symplectic manifolds of degree $n$ with zero $l_{1}$ are in one-to-one correspondence with twisted symplectic NQ-manifolds with $\left.\Theta\right|_{\mathcal{M}}=0$ (see Definition 3.11), to which the authors in [16] associate sigma models with boundary via AKSZ formalism.

The notion of a Lie bialgebroid was introduced by Mackenzie and Xu [25]. To study the double of a Lie bialgebroid, Liu-Weinstein-Xu introduced the notion of a Courant algebroid [23. Then Roytenberg gave an equivalent definition in [30. Symplectic NQ-manifolds of degree 2 are in one-to-one correspondence with Courant algebroids [28]. On the other hand, a Courant algebroid gives rise to a 2-term $L_{\infty}$-algebra [27. The present paper uses these facts to construct a new 2-term $L_{\infty}$-algebra from a given one as follows. Given a Lie 2-algebra $\mathfrak{g}=\mathfrak{g}_{0} \oplus \mathfrak{g}_{-1}$, its dual $\mathfrak{g}^{*}[1]$ is a homotopy Poisson manifold of degree 1 , which implies that $T^{*}[2] \mathfrak{g}^{*}[1]$ is a symplectic NQ-manifold of degree 2 . Thus we obtain a Courant algebroid $E=\mathfrak{g}_{-1}^{*} \times\left(\mathfrak{g}_{0} \oplus \mathfrak{g}_{0}^{*}\right)$, where $\mathfrak{g}_{-1}^{*}$ is the base manifold. Choosing some special sections of $E$

\footnotetext{
${ }^{1}$ There are different ways to describe the double of a Lie bialgebroid, e.g. Mackenzie gave the description of Drinfeld doubles for Lie bialgebroids using double Lie algebroids in 24]; Roytenberg and Voronov gave the description of Drinfeld doubles for Lie bialgebroids using graded manifolds in [28] and [39] respectively.
} 
and linear functions on the base manifold $\mathfrak{g}_{-1}^{*}$, we obtain a 2-term $L_{\infty}$-algebra $\tilde{\mathfrak{g}}=\tilde{\mathfrak{g}}_{0} \oplus \tilde{\mathfrak{g}}_{-1}$, where $\tilde{\mathfrak{g}}_{0}=\mathfrak{g}_{0} \oplus\left(\mathfrak{g}_{-1} \otimes \mathfrak{g}_{0}^{*}\right)$ and $\tilde{\mathfrak{g}}_{-1}=\mathfrak{g}_{-1}$. We thus have

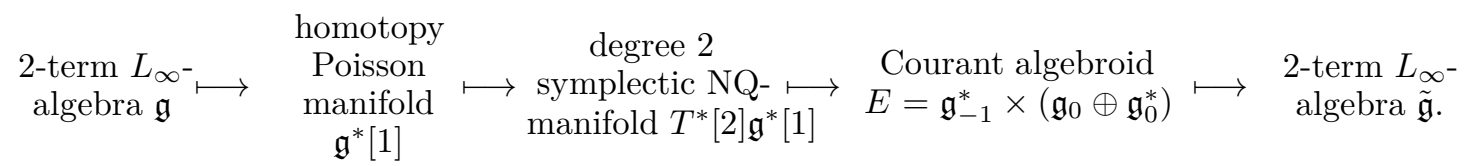

In particular, we obtain the 2-term $L_{\infty}$-algebra associated to the omni-Lie algebra [36, 41] from a vector space $V$, which is viewed as the abelian 2-term $L_{\infty}$-algebra $\left(V \stackrel{\text { id }}{\longrightarrow} V, l_{2}=0, l_{3}=0\right)$, and obtain the 2-term $L_{\infty}$-algebra of string type $\left[36\right.$ from the 2 -term $L_{\infty}$-algebra $\left(\mathbb{R} \stackrel{0}{\longrightarrow} \mathfrak{k}, l_{2}=[\cdot, \cdot]_{\mathfrak{k}}, l_{3}=0\right)$, where $\left(\mathfrak{k},[\cdot, \cdot]_{\mathfrak{k}}\right)$ is a Lie algebra. These interesting examples show that the construction of $\tilde{\mathfrak{g}}$ from $\mathfrak{g}$ has some important properties and applications, which will be studied in the future, since it is unrelated to the key subject of this paper. Nevertheless, we prove that there is a homomorphism from $\tilde{\mathfrak{g}}$ to $\mathfrak{g}$ (see Theorem 4.11).

We also observe that the Courant algebroid $E=\mathfrak{g}_{-1}^{*} \times\left(\mathfrak{g}_{0} \oplus \mathfrak{g}_{0}^{*}\right)$ obtained above is the double of a Lie quasi-bialgebroid $(A, \delta, \phi)$, where $A=\mathfrak{g}_{-1}^{*} \times \mathfrak{g}_{0}^{*} \longrightarrow \mathfrak{g}_{-1}^{*}$ is an action Lie algebroid. By integration, we get a Lie-quasi-Poisson groupoid $(\Gamma, \Pi, \phi)$, where $\Gamma=\mathfrak{g}_{-1}^{*} \times \mathfrak{g}_{0}^{*} \rightrightarrows \mathfrak{g}_{-1}^{*}$ is an action groupoid. In summary, we have

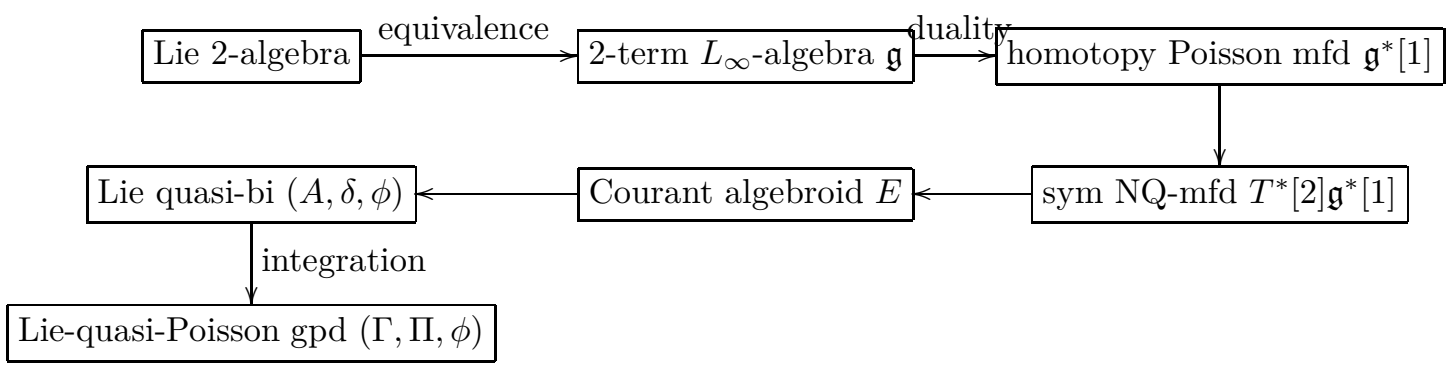

If we view the 2-vector space $\mathfrak{g}_{-1}^{*} \oplus \mathfrak{g}_{0}^{*} \rightrightarrows \mathfrak{g}_{-1}^{*}$ as the dual of the 2-vector space $\mathfrak{g}_{0} \oplus \mathfrak{g}_{-1} \rightrightarrows \mathfrak{g}_{0}$, this Lie-quasi-Poisson groupoid can be viewed as the natural geometric structure on the "dual" of a Lie 2-algebra.

Notations: For a graded vector space $V=\sum_{n \in \mathbb{Z}} V_{n}$, we use $V[l]$ to denote the $l$-shifted graded vector space, namely $V[l]_{n}=V_{l+n}$; we use $\operatorname{Sym}(V)$ to denote the symmetric algebra of $V$. We use $[\cdot, \cdot]_{S}$ to denote the Schouten bracket of sections of a Lie algebroid.

\section{Preliminaries}

Lie algebras can be categorified to Lie 2-algebras. For a good introduction on this subject see [4, 21]. Vector spaces can be categorified to 2-vector spaces. Let Vect be the category of vector spaces. A 2vector space is a category in the category Vect. A 2-vector space $C$ is a category with a vector space of objects $C_{0}$ and a vector space of morphisms $C_{1}$, such that all the structure maps are linear. Let $s, t: C_{1} \longrightarrow C_{0}$ be the source and target maps respectively.

The 2-category of 2-vector spaces is equivalent to the 2-category of 2-term complexes of vector spaces. Roughly speaking, given a 2-vector space $C, \operatorname{ker}(s) \stackrel{t}{\longrightarrow} C_{0}$ is a 2-term complex. Conversely, any 2-term complex of vector spaces $V_{1} \stackrel{\mathrm{d}}{\longrightarrow} V_{0}$ gives rise to a 2-vector space of which the set of objects is $V_{0}$, the set 
of morphisms is $V_{0} \oplus V_{1}$, the source map $s$ is given by $s(v+m)=v$, and the target map $t$ is given by $t(v+m)=v+\mathrm{d} m$, where $v \in V_{0}, m \in V_{1}$.

A Lie 2-algebra is a 2-vector space $C$ equipped with a skew-symmetric bilinear functor, such that the Jacobi identity is controlled by a natural isomorphism, which satisfies a coherence law of its own. The category of Lie 2-algebras is equivalent to the category of 2 -term $L_{\infty}$-algebras (see [4]).

Definition 2.1. An $L_{\infty}$-algebra is a graded vector space $\mathfrak{g}=\oplus_{i \in \mathbb{Z}} \mathfrak{g}_{i}$ equipped with a system $\left\{l_{k} \mid 1 \leq k<\right.$ $\infty\}$ of linear maps $l_{k}: \wedge^{k} \mathfrak{g} \longrightarrow \mathfrak{g}$ with degree $\operatorname{deg}\left(l_{k}\right)=2-k$, where the exterior powers are interpreted in the graded sense and the following relation with Koszul sign "Ksgn" is satisfied for all $n \geq 0$ :

$$
\sum_{i+j=n+1}(-1)^{i(j-1)} \sum_{\sigma} \operatorname{sgn}(\sigma) \operatorname{Ksgn}(\sigma) l_{j}\left(l_{i}\left(x_{\sigma(1)}, \cdots, x_{\sigma(i)}\right), x_{\sigma(i+1)}, \cdots, x_{\sigma(n)}\right)=0 .
$$

Here the summation is taken over all $(i, n-i)$-unshuffles with $i \geq 1$.

We denote an $L_{\infty}$-algebra by $\left(\oplus_{i \in \mathbb{Z}} \mathfrak{g}_{i},\left\{l_{i}\right\}_{i \geq 1}\right)$, or simply by $\mathfrak{g}$ if there is no confusion. In particular, we denote a 2 -term $L_{\infty}$-algebra by $\left(\mathfrak{g}_{-1} \stackrel{l_{1}}{\longrightarrow} \mathfrak{g}_{0}, l_{2}, l_{3}\right)$.

Definition 2.2. Let $\mathfrak{g}=\left(\mathfrak{g}_{-1} \stackrel{l_{1}}{\longrightarrow} \mathfrak{g}_{0}, l_{2}, l_{3}\right)$ and $\mathfrak{g}^{\prime}=\left(\mathfrak{g}_{-1}^{\prime} \stackrel{l_{1}^{\prime}}{\longrightarrow} \mathfrak{g}_{0}^{\prime}, l_{2}^{\prime}, l_{3}^{\prime}\right)$ be two 2-term $L_{\infty}$-algebras. An $L_{\infty}$-algebra homomorphism $F$ from $\mathfrak{g}$ to $\mathfrak{g}^{\prime}$ consists of: linear maps $F_{0}: \mathfrak{g}_{0} \rightarrow \mathfrak{g}_{0}^{\prime}, F_{1}: \mathfrak{g}_{-1} \rightarrow \mathfrak{g}_{-1}^{\prime}$ and $F_{2}: \mathfrak{g}_{0} \wedge \mathfrak{g}_{0} \rightarrow \mathfrak{g}_{-1}^{\prime}$, such that the following equalities hold for all $x, y, z \in \mathfrak{g}_{0}, m \in \mathfrak{g}_{-1}$,

(i) $F_{0} \circ l_{1}=l_{1}^{\prime} \circ F_{1}$,

(ii) $F_{0} l_{2}(x, y)-l_{2}^{\prime}\left(F_{0}(x), F_{0}(y)\right)=l_{1}^{\prime} F_{2}(x, y)$,

(iii) $F_{1} l_{2}(x, m)-l_{2}^{\prime}\left(F_{0}(x), F_{1}(m)\right)=F_{2}\left(x, l_{1}(m)\right)$,

(iv) $F_{2}\left(l_{2}(x, y), z\right)+c . p .+F_{1}\left(l_{3}(x, y, z)\right)=l_{2}^{\prime}\left(F_{0}(x), F_{2}(y, z)\right)+c . p .+l_{3}^{\prime}\left(F_{0}(x), F_{0}(y), F_{0}(z)\right)$.

Given a graded vector space $V=\sum_{n \in \mathbb{Z}} V_{n}$, it is well-known that there is a graded version of KosmannSchwarzbach's big bracket [18, which we denote by $\{\cdot, \cdot\}$, on $\operatorname{Sym}\left(V^{*}[l]\right) \otimes \operatorname{Sym}(V[k]) \cong \operatorname{Sym}\left(V^{*}[l] \oplus\right.$ $V[k]) \cong \operatorname{Sym}\left(T^{*}[l+k] V[k]\right)$ by extending the usual pairing between $V^{*}$ and $V$ via a graded Leibniz rule

$$
\begin{aligned}
\{u, v \wedge w\} & =\{u, v\} \wedge w+(-1)^{(|u|+l+k)|v|} v \wedge\{u, w\} \\
\{u, v\} & =-(-1)^{(|u|+k+l)(|v|+k+l)}\{v, u\}
\end{aligned}
$$

where $u \in \operatorname{Sym}\left(V^{*}[l] \oplus V[k]\right)_{|u|}$ and $v \in \operatorname{Sym}\left(V^{*}[l] \oplus V[k]\right)_{|v|}$. The big bracket is in fact the canonical graded Poisson bracket on $T^{*}[-l-k] V^{*}[-k]$. Thus, we have a graded Jacobi identity:

$$
\{u,\{v, w\}\}=\{\{u, v\}, w\}+(-1)^{(|u|+k+l)(|v|+k+l)}\{v,\{u, w\}\} .
$$

We view $l_{i}: \wedge^{i} \mathfrak{g} \longrightarrow \mathfrak{g}$ as elements in $\operatorname{Sym}^{i}\left(\mathfrak{g}^{*}[-1]\right) \otimes \mathfrak{g}[k]$. Then we have

Lemma 2.3. A series of degree $2-k$ elements $l_{i} \in\left(\operatorname{Sym}^{i}\left(\mathfrak{g}^{*}[-1]\right) \otimes \mathfrak{g}[k]\right)$ with $i=1,2, \ldots$ on $\mathfrak{g}=$ $\mathfrak{g}_{0} \oplus \mathfrak{g}_{-1} \oplus \ldots$ gives an $L_{\infty}$-algebra structure if and only if $\left\{\sum_{i=1}^{\infty} l_{i}, \sum_{i=1}^{\infty} l_{i}\right\}=0$.

Definition 2.4. A Courant algebroid is a vector bundle $E \longrightarrow M$, together with a fiber metric $(\cdot, \cdot)_{+}$ (so we can identify $E$ with $E^{*}$ ), a bundle map $\rho: E \longrightarrow T M$ (called the anchor), a bilinear bracket operation (Dorfman bracket) $\lceil\cdot, \cdot\rceil$ on $\Gamma(E)$, such that for all $e_{1}, e_{2}, e_{3} \in \Gamma(E)$, we have

$$
\begin{aligned}
\lceil e, e\rceil & =\frac{1}{2} D(e, e)_{+}, \\
\rho\left(e_{1}\right)\left(e_{2}, e_{3}\right)_{+} & =\left(\left\lceil e_{1}, e_{2}\right\rceil, e_{3}\right)_{+}+\left(e_{2},\left\lceil e_{1}, e_{3}\right\rceil\right)_{+}, \\
\left\lceil e_{1},\left\lceil e_{2}, e_{3}\right\rceil\right\rceil & =\left\lceil\left\lceil e_{1}, e_{2}\right\rceil, e_{3}\right\rceil+\left\lceil e_{2},\left\lceil e_{1}, e_{3}\right\rceil\right\rceil,
\end{aligned}
$$


where the operator $D: C^{\infty}(M) \longrightarrow \Gamma(E)$ is the map defined by

$$
(e, D f)_{+}=\rho(e) f
$$

One can also use the skew-symmetric Courant bracket $\llbracket \cdot, \cdot \rrbracket: \llbracket e_{1}, e_{2} \rrbracket=\frac{1}{2}\left(\left\lceil e_{1}, e_{2}\right\rceil-\left\lceil e_{2}, e_{1}\right\rceil\right)$. But the equality (7) does not hold anymore. We have

$$
\llbracket \llbracket e_{1}, e_{2} \rrbracket, e_{3} \rrbracket+\llbracket \llbracket e_{2}, e_{3} \rrbracket, e_{1} \rrbracket+\llbracket \llbracket e_{3}, e_{1} \rrbracket, e_{2} \rrbracket=D T\left(e_{1}, e_{2}, e_{3}\right),
$$

where $T\left(e_{1}, e_{2}, e_{3}\right) \in C^{\infty}(M)$ is defined by

$$
T\left(e_{1}, e_{2}, e_{3}\right)=\frac{1}{6}\left(\llbracket e_{1}, e_{2} \rrbracket, e_{3}\right)_{+}+c . p . .
$$

Theorem 2.5. (27]) A Courant algebroid structure on a vector bundle $E$ gives rise to a 2-term $L_{\infty}$ algebra structure on $C^{\infty}(M) \oplus \Gamma(E)$, where $l_{i}$ are given by

$$
\left\{\begin{aligned}
l_{1}(f) & =D f, & & \forall f \in C^{\infty}(M), \\
l_{2}\left(e_{1}, e_{2}\right) & =\llbracket e_{1}, e_{2} \rrbracket & & \forall e_{1}, e_{2} \in \Gamma(E), \\
l_{2}\left(e_{1}, f\right) & =\frac{1}{2}\left(e_{1}, D f\right)_{+} & & \forall e_{1} \in \Gamma(E), f \in C^{\infty}(M), \\
l_{3}\left(e_{1}, e_{2}, e_{3}\right) & =-T\left(e_{1}, e_{2}, e_{3}\right) & & \forall e_{1}, e_{2}, e_{3} \in \Gamma(E) .
\end{aligned}\right.
$$

A Courant algebroid can be described by a symplectic NQ-manifold of degree 2 [28. Explicitly, let $\left(E,(\cdot, \cdot)_{+}\right)$be a pseudo-Euclidean vector bundle over a manifold $M$. Then, $E[1]$ is a Poisson N-manifold of degree -2 . Let $\mathcal{M}$ be its minimal symplectic realization. An NQ-structure on $\mathcal{M}$ is determined by a cubic Hamiltonian function $\Theta$ on $\mathcal{M}$ satisfying $\{\Theta, \Theta\}=0$, where $\{\cdot, \cdot\}$ is the even Poisson bracket on $\mathcal{M}$ of degree -2 . Such a $\Theta$ corresponds to a Courant algebroid structure on $E$. In fact, we define the anchor $\rho$ and the Dorfman bracket $\lceil\cdot, \cdot]$ as the derived bracket by

$$
\rho(e) f=\{\{e, \Theta\}, f\}, \quad\left\lceil e_{1}, e_{2}\right\rceil=\left\{\left\{e_{1}, \Theta\right\}, e_{2}\right\}, \quad \forall f \in C^{\infty}(M), \forall e, e_{1}, e_{2} \in \Gamma(E) .
$$

Here $C^{\infty}(M)$ and $\Gamma(E)$ are the algebras of degree 0 and degree 1 polynomial functions on $\mathcal{M}$ respectively. We refer to [39] for a general construction of doubles for graded QS-manifolds and QP-manifolds.

For a vector bundle $A$, consider the graded manifold $T^{*}[2] A[1]$. It is canonically equipped with a Poisson bracket of degree -2 , and is actually the minimal symplectic realization of $A \oplus A^{*}$. This Poisson bracket, called the big bracket in [18, is denoted here by $\{\cdot, \cdot\}$. Let $\left(x^{i}, \xi^{a}\right)$ be local coordinates on $A[1]$, we denote by $\left(x^{i}, \xi^{a}, \theta_{a}, p_{i}\right)$ the local coordinates on $T^{*}[2] A[1]$. About their degrees, we have

$$
\operatorname{degree}\left(x^{i}, \xi^{a}, \theta_{a}, p_{i}\right)=(0,1,1,2) .
$$

The big bracket satisfies

$$
\left\{x^{i}, p_{j}\right\}=\delta_{j}^{i}=-\left\{p_{j}, x^{i}\right\}, \quad\left\{\xi^{a}, \theta_{b}\right\}=\delta_{j}^{i}=\left\{\theta_{b}, \xi^{a}\right\} .
$$

A Lie algebroid structure on $A$ is equivalent to a degree 3 function $\mu=\rho_{b}^{i} p_{i} \xi^{b}+\frac{1}{2} \mu_{b c}^{a} \xi^{b} \xi^{c} \theta_{a}$ such that $\{\mu, \mu\}=0$.

A Lie quasi-bialgebroid structure on $A$ is given by a degree 3 function $\mu+\gamma+\phi$, which can be locally written as

$$
\mu=\rho_{b}^{i} p_{i} \xi^{b}+\frac{1}{2} \mu_{b c}^{a} \xi^{b} \xi^{c} \theta_{a}, \quad \gamma=\varrho^{i b} p_{i} \theta_{b}+\frac{1}{2} \gamma_{a}^{b c} \xi^{a} \theta_{b} \theta_{c}, \quad \phi=\frac{1}{6} \phi^{a b c} \theta_{a} \theta_{b} \theta_{c},
$$


and they satisfy

$$
\{\mu+\gamma+\phi, \mu+\gamma+\phi\}=0 .
$$

More precisely, we have

$$
\{\mu, \mu\}=0, \quad\{\mu, \gamma\}=0, \quad \frac{1}{2}\{\gamma, \gamma\}+\{\mu, \phi\}=0, \quad\{\gamma, \phi\}=0 .
$$

See [19] for more details. On $A \oplus A^{*}$, there is a natural Courant algebroid structure, in which the degree 3 function $\Theta$ is exactly $\mu+\gamma+\phi$. Note that there are two canonical fiber metric $(\cdot, \cdot)_{ \pm}$on $A \oplus A^{*}$ :

$$
(x+\xi, y+\eta)_{ \pm}=\langle\xi, y\rangle \pm\langle x, \eta\rangle,
$$

where $\langle\cdot, \cdot\rangle$ is the usual pairing without any degree involved in.

\section{Maurer-Cartan elements on homotopy Poisson manifolds}

First we recall the notions of homotopy Poisson algebras and homotopy Poisson manifolds. They are also called $P_{\infty}$-manifolds in [10, 32] and higher Poisson manifolds in [8, 17, 40]. Here we follow the convention in [26].

Definition 3.1. A homotopy Poisson algebra of degree $n$ is a graded commutative algebra a over a field of characteristic zero with an $L_{\infty}$-algebra structure $\left\{l_{m}\right\}_{m \geq 1}$ on $\mathfrak{a}[n]$, such that the map

$$
x \longrightarrow l_{m}\left(x_{1}, \cdots, x_{m-1}, x\right), \quad x_{1}, \cdots, x_{m-1}, x \in \mathfrak{a}
$$

is a derivation of $\mathfrak{a}$ of degree $\kappa:=2-m-n(m-1)+\sum_{i=1}^{m-1}\left|x_{i}\right|$, i.e., for all $x, y \in \mathfrak{a}$,

$$
l_{m}\left(x_{1}, \cdots, x_{m-1}, x y\right)=l_{m}\left(x_{1}, \cdots, x_{m-1}, x\right) y+(-1)^{\kappa|x|} x l_{m}\left(x_{1}, \cdots, x_{m-1}, y\right) .
$$

Here, $|x|$ denotes the degree of $x \in \mathfrak{a}$.

A homotopy Poisson algebra of degree $n$ is of finite type if there exists a $q$ such that $l_{m}=0$ for all $m>q$.

$A$ homotopy Poisson manifold of degree $n$ is a graded manifold $\mathcal{M}$ whose algebra of functions $C^{\infty}(\mathcal{M})$ is equipped with a degree $n$ homotopy Poisson algebra structure of finite type.

Throughout this paper, we use $\left(\mathcal{M},\left\{l_{i}\right\}_{1 \leq i<\infty}\right)$ to denote a homotopy Poisson manifold. Obviously, a usual Poisson manifold is a homotopy Poisson manifold of degree 0 .

Remark 3.2. In this remark, we compare the related structures in the literature.

(i) In [10], the authors introduced the notion of a $P_{\infty}$-algebra, which is a graded commutative algebra $\mathfrak{a}$ with an $L_{\infty}$-algebra structure such that the Leibniz rule is satisfied. So a $P_{\infty}$-algebra is a homotopy Poisson algebra of degree 0.

(ii) The notion of a higher Poisson structure was introduced in [40], and further studied in [8, 17], where the authors used the superized version of an $L_{\infty}$-algebra, i.e., $\mathbb{Z}_{2}$-graded.

(iii) A graded Poisson algebra of degree $k$ is a graded commutative algebra a with a degree - $k$ Lie bracket, such that the bracket is a biderivation of the product, namely

$$
[x, y \cdot z]=[x, y] \cdot z+(-1)^{|y|(|x|+k)} y \cdot[x, z] .
$$

See [9] for more details. Thus, a graded Poisson algebra of degree $k$ is a homotopy Poisson algebra of degree $k$. In particular, the associated $L_{\infty}$-algebra has only one non-zero map $l_{2}$. 
In the following, we list a few interesting examples of different kinds.

Example 3.3. Let $A$ be a Lie algebroid. Consider its dual vector bundle, $A^{*}[1]$, which is an N-manifold of degree 1. Its algebra of polynomial functions is

$$
\cdots \oplus \Gamma(A) \oplus C^{\infty}(M)
$$

where $\Gamma(A)$ is of degree 1 , and $C^{\infty}(M)$ is of degree 0 . The Poisson bracket is in fact the Schouten bracket $[\cdot, \cdot]_{S}$ on $\Gamma\left(\wedge^{\bullet} A\right)$. It is straightforward to see that $A^{*}[1]$ is a homotopy Poisson manifold of degree 1.

Example 3.4. For an arbitrary manifold $M$, the shifted cotangent bundle $T^{*}[1] M$ is a symplectic Nmanifold of degree 1 (see [11, 28]). Its algebra of polynomial functions is

$$
\cdots \oplus \mathfrak{X}(M) \oplus C^{\infty}(M),
$$

where $\mathfrak{X}(M)$ is of degree 1 , and $C^{\infty}(M)$ is of degree 0 . The Poisson bracket is exactly the Schouten bracket $[\cdot, \cdot]_{S}$ on $\Gamma\left(\wedge^{\bullet} T M\right)$. It is straightforward to see that $\left(T^{*}[1] M, l_{2}=[\cdot, \cdot]_{S}\right)$ is a homotopy Poisson manifold of degree 1. Similarly, any symplectic N-manifold of degree $n$ is a homotopy Poisson manifold of degree $n$. The $L_{\infty}$-algebra structure here only contains one nonzero $l_{2}$.

Example 3.5. Given a 2 -term $L_{\infty}$-algebra $\mathfrak{g}=\left(\mathfrak{g}_{-1} \stackrel{l_{1}}{\longrightarrow} \mathfrak{g}_{0}, l_{2}, l_{3}\right)$, its graded dual space $\mathfrak{g}^{*}[1]=$ $\mathfrak{g}_{0}^{*}[1] \oplus \mathfrak{g}_{-1}^{*}[1]$ is an N-manifold of degree 1 with the base manifold $\mathfrak{g}_{-1}^{*}$. Its algebra of polynomial functions is

$$
\cdots \oplus\left(C^{\infty}\left(\mathfrak{g}_{-1}^{*}\right) \otimes \mathfrak{g}_{0}\right) \oplus C^{\infty}\left(\mathfrak{g}_{-1}^{*}\right) .
$$

There is a degree 1 homotopy Poisson algebra structure on it obtained by extending the original 2-term $L_{\infty}$-algebra structure using the Leibniz rule. Thus, the dual of a 2 -term $L_{\infty}$-algebra is a homotopy Poisson manifold of degree 1. This generalizes the fact that the dual of a Lie algebra is a linear Poisson manifold.

Similarly, the dual of an $n$-term $L_{\infty}$-algebra is a homotopy Poisson manifold of degree $n-1$.

Example 3.6. Given a splitting Lie $n$-algebroid $2 \mathcal{A}=A_{0} \oplus \cdots \oplus A_{1-n}$, its dual $\mathcal{A}^{*}[n]=A_{0}^{*}[n] \oplus \cdots \oplus$ $A_{1-n}^{*}[n]$ is an N-manifold of degree $n$. Its algebra of polynomial functions is

$$
\cdots \oplus\left(\Gamma\left(A_{0}\right) \oplus \Gamma\left(\wedge^{n} A_{1-n}\right) \oplus \cdots\right) \oplus \cdots \oplus \Gamma\left(A_{1-n}\right) \oplus C^{\infty}(M) .
$$

There is a degree $n$ homotopy Poisson algebra structure on it. Thus, the dual of a splitting Lie $n$-algebroid is a homotopy Poisson manifold of degree $n$.

It is known that $(M, \pi)$ is a Poisson manifold if and only if $\left(T^{*}[1] M, Q\right)$ is a symplectic NQ-manifold of degree 1, where the homological vector field $Q$ is given by $Q=\{\pi, \cdot\}$. More generally, the cotangent bundle of a homotopy Poisson manifold of degree $n$ gives rise to a symplectic NQ-manifold of degree $n+1$. Consider the homotopy Poisson manifold given in Example 3.5, we have

Proposition 3.7. Given an n-term $L_{\infty}$-algebra $\mathfrak{g}=\left(\mathfrak{g}_{0} \oplus \cdots \oplus \mathfrak{g}_{-n+1},\left\{l_{i}\right\}_{1 \leq i \leq n+1}\right)$, the cotangent bundle $T^{*}[n] \mathfrak{g}^{*}[n-1]$ is a symplectic NQ-manifold of degree $n$, where the degree 1 homological vector field $Q$ is given by

$$
Q=\left\{\sum l_{i}, \cdot\right\}
$$

in which $\{\cdot, \cdot\}$ is the canonical Poisson structure, and $\sum l_{i} \in \operatorname{Sym}\left(\mathfrak{g}^{*}[-1]\right) \otimes \mathfrak{g}[1-n]$ is viewed as a polynomial function of degree $n+1$ on $T^{*}[n] \mathfrak{g}^{*}[n-1]$.

\footnotetext{
${ }^{2}$ By definition, there is an $L_{\infty}$-algebra structure on the complex of section spaces together with some compatibility conditions. See [7] 35] for more details.
} 
Similar to 13, a Maurer-Cartan element on a degree $n$ homotopy Poisson manifold $\mathcal{M}$ is given by a function $\alpha$ on $\mathcal{M}$ satisfying the Maurer-Cartan equation

$$
\sum_{i} \frac{(-1)^{i}}{i !} l_{i}(\alpha, \cdots, \alpha)=0 .
$$

Example 3.8. (quasi-Poisson $\mathfrak{g}$-manifolds) Let $M$ be a manifold and $\left(\mathfrak{k},[\cdot, \cdot]_{\mathfrak{k}}, K\right)$ a quadratic Lie algebra. Let $\left\{e_{a}\right\}$ be an orthogonal basis of $\mathfrak{k}$ with respect to the metric $K$, i.e. $K\left(e_{a}, e_{b}\right)=\delta_{a b}$, and $\left\{e^{a}\right\}$ its dual basis. Then $K$ induces an isomorphism, which we denote by $K^{\sharp}$, from $\mathfrak{k}^{*}$ to $\mathfrak{k}^{\mathfrak{k}}$ via $K\left(K^{\sharp}(\xi), u\right)=\langle\xi, u\rangle$. More precisely, we have $K^{\sharp}\left(e^{a}\right)=e_{a}$. We define $R \in \wedge^{3} \mathfrak{k}^{*}$ by

$$
R(u, v, w)=K\left([u, v]_{\mathfrak{k}}, w\right), \quad \forall u, v, w \in \mathfrak{k} .
$$

Then $\mathcal{M}:=T^{*}[1] M \times \mathfrak{k}[1]$ is a homotopy Poisson manifold of degree 1 . In fact, we can define an $L_{\infty}$-algebra structure on $C^{\infty}(\mathcal{M})$ generated by

$$
l_{2}(X, f)=X f, \quad l_{2}(X, Y)=[X, Y]_{S}, \quad l_{1}(\xi)=\delta(\xi), \quad l_{3}(\xi, \eta, \gamma)=K^{\sharp}(R)(\xi, \eta, \gamma),
$$

where $f \in C^{\infty}(M), X, Y \in \mathfrak{X}(M), \xi, \eta, \gamma \in \mathfrak{k}^{*}$, and $\delta: \wedge^{\bullet \mathfrak{k}^{*}} \longrightarrow \wedge^{\bullet+1} \mathfrak{k}^{*}$ is the coboundary operator associated to the Lie algebra $\mathfrak{k}$.

A degree 2 function $\alpha=\pi+\rho$, where $\pi \in \wedge^{2} \mathfrak{X}(M)$ and $\rho \in \mathfrak{k}^{*} \otimes \mathfrak{X}(M)$, is a Maurer-Cartan element, i.e. $-l_{1}(\alpha)+\frac{1}{2} l_{2}(\alpha, \alpha)-\frac{1}{3 !} l_{3}(\alpha, \alpha, \alpha)=0$, if and only if the following three conditions hold:

$$
l_{1}(\rho)=\frac{1}{2}[\rho, \rho]_{S}, \quad[\pi, \rho]_{S}=0, \quad \frac{1}{2}[\pi, \pi]_{S}=\frac{1}{6} K^{\sharp}(R)(\rho, \rho, \rho) .
$$

These conditions are equivalent to that $\rho: \mathfrak{k} \longrightarrow \mathfrak{X}(M)$ is a Lie algebra morphism, $\pi$ is $\mathfrak{k}$-invariant and $\frac{1}{2}[\pi, \pi]_{S}=\wedge^{3} \rho\left(K^{\sharp}(R)\right)$ respectively. Therefore, a quasi-Poisson $\mathfrak{g}$-manifold [1, 2] gives rise to a Maurer-Cartan element on $\mathcal{M}$.

A homotopy Poisson manifold $\left(\mathcal{M},\left\{l_{i}\right\}_{1 \leq i<\infty}\right)$ is called symplectic if the binary bracket $l_{2}$ is nondegenerate. We refer the reader to [17] for a thorough discussion of homotopy symplectic structures in the setting of $\mathbb{Z}_{2}$-graded manifolds.

We turn to study Maurer-Cartan elements on a homotopy symplectic manifold of degree $n$. First we present some examples with mathematical and physical interests.

Example 3.9. (twisted Poisson structures) The shifted cotangent bundle $T^{*}[1] M$ of a manifold $M$ is canonically a symplectic $N$-manifold of degree 1. Slightly different from Example 3.4, with a choice of a closed 3 -form $H$, we introduce a nontrivial $l_{3}$ on the algebra of functions of $T^{*}[1] M$ by $l_{3}(X, Y, Z)=H(X, Y, Z)$, for all $X, Y, Z \in \mathfrak{X}(M)$. The compatibility of $l_{2}$ and $l_{3}$ is due to the fact that $H$ is closed. Thus, $\left(T^{*}[1] M, l_{2}=[\cdot, \cdot]_{S}, l_{3}=H\right)$ is a homotopy symplectic manifold of degree 1 . The idea of adding a closed 3 -form to obtain a new $L_{\infty}$-algebra was first introduced in [31.

A degree 2 function $\pi$ is a Maurer-Cartan element of $T^{*}[1] M$ if and only if

$$
\frac{1}{2} l_{2}(\pi, \pi)-\frac{1}{3 !} l_{3}(\pi, \pi, \pi)=0,
$$

which is equivalent to $\frac{1}{2}[\pi, \pi]=\wedge^{3} \pi^{\sharp} H$, that is, $\pi$ is a twisted Poisson structure $([29$, 34] $)$ on $M$. 
Example 3.10. (twisted Courant algebroids) Let $E \longrightarrow M$ be a vector bundle with a fiber metric $(\cdot, \cdot)_{+}$, and $H$ a closed 4 -form on $M$. Let $\mathcal{M}$ be its minimal symplectic realization. See [28] for details. Then $\mathcal{A}^{2}$ is the section space of a vector bundle $\mathbb{A}$, which fits in the following exact sequence:

$$
0 \longrightarrow \wedge^{2} E^{*} \longrightarrow \mathbb{A} \stackrel{a}{\longrightarrow} T M \longrightarrow 0
$$

Similar to the treatment in Example 3.9. we can define a new degree 2 homotopy Poisson algebra structure on the algebra of functions of $\mathcal{M}$ by adding

$$
l_{4}\left(\chi_{1}, \chi_{2}, \chi_{3}, \chi_{4}\right)=H\left(a\left(\chi_{1}\right), a\left(\chi_{2}\right), a\left(\chi_{3}\right), a\left(\chi_{4}\right)\right), \quad \forall \chi_{i} \in \mathcal{A}^{2} .
$$

Choose a local coordinate $\left(x^{i}, p_{i}, \xi^{a}\right)$. A degree 3 function $\alpha=\rho_{a}^{i} p_{i} \xi^{a}-\frac{1}{3 !} f_{a b c} \xi^{a} \xi^{b} \xi^{c}$ is a MaurerCartan element if and only if $\frac{1}{2} l_{2}(\alpha, \alpha)+\frac{1}{24} l_{4}(\alpha, \alpha, \alpha, \alpha)=0$. Define an anchor $\rho: E \rightarrow T M$ and a derived bracket $\lceil\cdot, \cdot\rceil$ on $\Gamma(E)$ by

$$
\rho(e) f=l_{2}\left(l_{2}(e, \alpha), f\right), \quad\left\lceil e_{1}, e_{2}\right\rceil=l_{2}\left(l_{2}\left(e_{1}, \alpha\right), e_{2}\right) .
$$

According to [28, Theorem 4.5], we have

$$
\begin{aligned}
l_{2}(\alpha, \alpha)= & \left(\rho^{*}\left(d x^{i}\right), \rho^{*}\left(d x^{j}\right)\right)_{+} p_{i} p_{j}+\xi^{a} \xi^{b} d x^{j}\left(\left[\rho\left(e_{a}\right), \rho\left(e_{b}\right)\right]_{S}-\rho\left(\left\lceil e_{a}, e_{b}\right\rceil\right)\right) p_{j} \\
& +\frac{1}{12}\left(\left\lceil\left\lceil e_{a}, e_{b}\right\rceil, e_{c}\right\rceil+\left\lceil e_{b},\left\lceil e_{a}, e_{c}\right\rceil\right\rceil-\left\lceil e_{a},\left\lceil e_{b}, e_{c}\right\rceil\right\rceil, e_{d}\right)_{+} \xi^{a} \xi^{b} \xi^{c} \xi^{d}
\end{aligned}
$$

On the other hand, a straightforward calculation gives that

$$
l_{4}(\alpha, \alpha, \alpha, \alpha)=\rho^{*} H
$$

Thus, the condition for that $\alpha$ is a Maurer-Cartan element is equivalent to that $(E, K, \rho,\lceil\cdot, \cdot\rceil, H)$ is a twisted Courant algebroid, which arises from the study of three dimensional sigma models with WessZumino term [14]. See [42] for its close relation with coisotropic Cartan geometry.

We have seen that the study of a general homotopy symplectic manifold of degree $n$ and its MaurerCartan elements is inspired by many interesting geometric structures. Next, we will see that these structures naturally appear in the study of topological field theory. To be precise, it is known that there exists a systematic method to construct a topological sigma model from a symplectic NQ-manifold, which is called the Alexandrov-Kontsevich-Schwartz-Zaboronsky (AKSZ) formalism [3]. We will show that this construction can be extended to the case of a degree $n$ homotopy symplectic manifold $\left(\mathcal{M},\left\{l_{i}\right\}_{2 \leq i<\infty}\right)$ with a degree $n+1$ Maurer-Cartan element $\alpha$. To do this, we need the notion of a twisted symplectic NQ-manifold, which was introduced in [16] to describe the structure on the target supermanifold of the AKSZ sigma model with boundary.

Definition 3.11. [16] Let $\left(\mathcal{M},\{\cdot, \cdot\}_{s}\right)$ be a symplectic $N$-manifold of degree $n$ and $\alpha$ a degree $n+1$ function on it. Then $\left(\mathcal{M},\{\cdot, \cdot\}_{s}, \alpha\right)$ is called a twisted symplectic $N Q$-manifold if there is a degree $n+1$ symplectic $N Q$-manifold $\left(T^{*}[n+1] \mathcal{M},\{\cdot, \cdot\}, \Theta\right)$ such that

(a) $\{\cdot, \cdot\}_{s}=\left.\{\cdot,\{\cdot, \Theta\}\}\right|_{\mathcal{M}}$

(b) it satisfies the canonical transformation equation $\left.e^{-\alpha} \Theta\right|_{\mathcal{M}}=0$, where

$$
e^{-\alpha} \Theta=\Theta-\{\alpha, \Theta\}+\frac{1}{2}\{\alpha,\{\alpha, \Theta\}\}-\cdots
$$


The following theorem states that a homotopy symplectic manifold with a Maurer-Cartan element naturally gives rise to a twisted symplectic NQ-manifold.

Theorem 3.12. Degree $n$ homotopy symplectic manifolds $\left(\mathcal{M},\left\{l_{i}\right\}_{2 \leq i<\infty}\right)$ with a degree $n+1$ MaurerCartan element $\alpha$ are in one-to-one correspondence with twisted symplectic $N Q$-manifolds $\left(\mathcal{M},\{\cdot, \cdot\}_{s}, \alpha\right)$ with $\left.\Theta\right|_{\mathcal{M}}=0$.

Proof. Let $\left(\mathcal{M},\left\{l_{i}\right\}_{2 \leq i<\infty}\right)$ be a homotopy symplectic manifold of degree $n$ and $\alpha$ a degree $n+1$ MaurerCartan element. Then $\left(T^{*}[n+1] \mathcal{M},\{\cdot, \cdot\}, \Theta=\sum l_{i}\right)$ is a symplectic NQ-manifold of degree $n+1$, and the following equality holds:

$$
l_{k}\left(a_{1}, \cdots, a_{k}\right)=\left.\left\{a_{k}, \cdots,\left\{a_{2},\left\{a_{1}, \sum l_{i}\right\}\right\} \cdots\right\}\right|_{\mathcal{M}} .
$$

Define $\{\cdot, \cdot\}_{s}=l_{2}$, which is a Poisson bracket determined by a nondegenerate closed 2-form. Now we extend $\alpha$ to a function on $T^{*}[n+1] \mathcal{M}$, which is constant along the fiber. By (13), we have $\left.\Theta\right|_{\mathcal{M}}=$ $\left.\{\alpha, \Theta\}\right|_{\mathcal{M}}=0$ and

$$
\left.e^{-\alpha} \Theta\right|_{\mathcal{M}}=\left.\frac{1}{2}\{\alpha,\{\alpha, \Theta\}\}\right|_{\mathcal{M}}-\left.\frac{1}{6}\{\alpha,\{\alpha,\{\alpha, \Theta\}\}\}\right|_{\mathcal{M}}+\cdots=\frac{1}{2} l_{2}(\alpha, \alpha)-\frac{1}{3 !} l_{3}(\alpha, \alpha, \alpha)+\cdots .
$$

Thus, the condition for that $\alpha$ satisfies the Maurer-Cartan equation on $\left(\mathcal{M},\left\{l_{i}\right\}_{2 \leq i<\infty}\right)$ is equivalent to that the canonical transformation equation $\left.e^{-\alpha} \Theta\right|_{\mathcal{M}}=0$ holds on $T^{*}[n+1] \mathcal{M}$.

One immediate consequence of the main construction in [16] is that, associated to a degree $n$ homotopy symplectic manifold $\left(\mathcal{M},\left\{l_{i}\right\}_{2 \leq i<\infty}\right)$ with a degree $n+1$ Maurer-Cartan element $\alpha$, there is an AKSZ sigma model with boundary. This can be viewed as a generalization of AKSZ formalism to the setting of homotopy symplectic manifolds. In particular, if $\left(\mathcal{M},\left\{l_{i}\right\}_{2 \leq i<\infty}\right)$ is a symplectic NQ-manifold, i.e. $l_{i}=0$ for $i \geq 3$, it becomes the AKSZ construction.

\section{2-term $L_{\infty}$-algebras and Courant algebroids}

In this section, we obtain a Courant algebroid from a 2 -term $L_{\infty}$-algebra. Consequently, we give a general construction of a 2 -term $L_{\infty}$-algebra from a 2 -term $L_{\infty}$-algebra, which could produce many interesting examples including the 2 -term $L_{\infty}$-algebra associated to an omni-Lie algebra and the 2 -term $L_{\infty}$-algebra of string type.

Let $\mathfrak{g}=\left(\mathfrak{g}_{-1} \stackrel{l_{1}}{\longrightarrow} \mathfrak{g}_{0}, l_{2}=l_{2}^{0}+l_{2}^{1}, l_{3}\right)$ be a 2 -term $L_{\infty}$-algebra, where

$$
l_{1} \in \mathfrak{g}_{-1}^{*} \otimes \mathfrak{g}_{0}, \quad l_{2}^{0} \in \wedge^{2} \mathfrak{g}_{0}^{*} \otimes \mathfrak{g}_{0}, \quad l_{2}^{1} \in \mathfrak{g}_{0}^{*} \wedge \mathfrak{g}_{-1}^{*} \otimes \mathfrak{g}_{-1}, \quad l_{3} \in \wedge^{3} \mathfrak{g}_{0}^{*} \otimes \mathfrak{g}_{-1} .
$$

By Proposition 3.7, the cotangent bundle $T^{*}[2] \mathfrak{g}^{*}[1]$ is a symplectic NQ-manifold of degree 2. Now the canonical Poisson structure $\{\cdot, \cdot\}$ is given by (2) -(4), in which $k=l=-1$. We give the relation between $l_{i}$ and $\{\cdot, \cdot\}$ by the following lemma.

Lemma 4.1. For all $x, y \in \mathfrak{g}_{0}$ and $m \in \mathfrak{g}_{-1}$, we have

$$
\begin{aligned}
l_{1}(m) & =\left\{m, l_{1}\right\}=-\left\{l_{1}, m\right\}, \\
l_{2}^{0}(x, y) & =\left\{y,\left\{x, l_{2}^{0}\right\}\right\}=-\left\{\left\{x, l_{2}^{0}\right\}, y\right\} \\
l_{2}^{1}(x, m) & =\left\{m,\left\{x, l_{2}^{1}\right\}\right\}=-\left\{\left\{x, l_{2}^{1}\right\}, m\right\}, \\
l_{3}(x, y, \cdot) & =\left\{y,\left\{x, l_{3}\right\}\right\}=-\left\{\left\{x, l_{3}\right\}, y\right\} .
\end{aligned}
$$


On the other hand, symplectic NQ-manifolds of degree 2 are in one-to-one correspondence with Courant algebroids [28]. Thus, from $T^{*}[2] \mathfrak{g}^{*}[1]$, we obtain a Courant algebroid $E$ :

$$
E=\mathfrak{g}_{-1}^{*} \times\left(\mathfrak{g}_{0}^{*} \oplus \mathfrak{g}_{0}\right) \longrightarrow \mathfrak{g}_{-1}^{*},
$$

in which the anchor and the Dorfman bracket are defined by the derived bracket using the degree 3 function $l=-\sum l_{i}$. Note that $E$ is the direct sum of two vector bundles $A$ and $A^{*}$, where $A=\mathfrak{g}_{-1}^{*} \times \mathfrak{g}_{0}^{*} \longrightarrow \mathfrak{g}_{-1}^{*}$ and $A^{*}=\mathfrak{g}_{-1}^{*} \times \mathfrak{g}_{0} \longrightarrow \mathfrak{g}_{-1}^{*}$. The fiber metric is given by the canonical pairing between $A$ and $A^{*}$.

It is necessary to give the precise structures on the Courant algebroid $E$.

Proposition 4.2. Consider the Courant algebroid $E$ given above. For constant sections $x, y \in \mathfrak{g}_{0}, \xi, \eta \in$ $\mathfrak{g}_{0}^{*}$ and a linear function $m \in \mathfrak{g}_{-1}$, we have

(i) the anchor of $x$ is a linear vector field, more precisely, $\rho(x)(m)=l_{2}^{1}(x, m)$;

(ii) the anchor of $\xi$ is a constant vector field, more precisely, $\rho(\xi)=-l_{1}^{*}(\xi)$, where $l_{1}^{*}$ is defined by $\left\langle l_{1}^{*}(\xi), m\right\rangle=-\left\langle\xi, l_{1}(m)\right\rangle$;

(iii) the image of a linear function under the operator $D$ is not a constant section, we have

$$
D m=l_{1}(m)-l_{2}^{1}(m, \cdot) \in \mathfrak{g}_{0}+\mathfrak{g}_{0}^{*} \otimes \mathfrak{g}_{-1}
$$

(iv) the constant sections in $\mathfrak{g}_{0}$ under the Dorfman bracket are not closed, but we have

$$
\lceil x, y\rceil=l_{2}^{0}(x, y)+l_{3}(x, y, \cdot) \in \mathfrak{g}_{0}+\mathfrak{g}_{0}^{*} \otimes \mathfrak{g}_{-1} ;
$$

(v) the Dorfman bracket of two constant sections in $\mathfrak{g}_{0}^{*}$ is zero, i.e. $\lceil\xi, \eta\rceil=0$;

(vi) the Dorfman bracket of a constant section in $\mathfrak{g}_{0}^{*}$ and a constant section in $\mathfrak{g}_{0}$ is a constant section in $\mathfrak{g}_{0}^{*}$,

$$
\lceil x, \xi\rceil=-\lceil\xi, x\rceil=l_{2}^{0}(x, \cdot)^{*} \xi \in \mathfrak{g}_{0}^{*},
$$

where $l_{2}^{0}(x, \cdot) \in \mathfrak{g l}\left(\mathfrak{g}_{0}\right)$ can be viewed as the adjoint map, and $l_{2}^{0}(x, \cdot)^{*}$ is its dual map.

Proof. By the relation $\rho(x)(m)=\{\{x,-l\}, m\}$ and Lemma 4.1, (i) is obvious. (ii) follows from

$$
\begin{aligned}
\rho(\xi)(m) & =\{\{\xi,-l\}, m\}=\left\{\left\{\xi,-l_{1}\right\}, m\right\}=-\left\{\xi,\left\{l_{1}, m\right\}\right\} \\
& =\left\{\xi,\left\{m, l_{1}\right\}\right\}=\left\{\xi, l_{1}(m)\right\}=-\left\langle l_{1}^{*}(\xi), m\right\rangle .
\end{aligned}
$$

(iii) follows from the relation $D m=\{-l, m\}$ and Lemma 4.1. (iv)-(vi) also follow from the relation $\lceil a, b\rceil=\{\{a,-l\}, b\}$ for all sections $a, b$ and Lemma 4.1 .

We have seen that we can obtain linear sections through the Dorfman bracket of constant sections. Thus, it is not enough if we only consider constant sections.

Corollary 4.3. Let $x \in \mathfrak{g}_{0}$ be a constant section, and $\phi=\xi \otimes m, \psi=\eta \otimes n: \mathfrak{g}_{-1}^{*} \longrightarrow \mathfrak{g}_{0}^{*}$ be linear sections, where $\xi, \eta \in \mathfrak{g}_{0}^{*}$ and $m, n \in \mathfrak{g}_{-1}$. Then we have

(i) $\lceil x, \xi \otimes m\rceil=\xi \otimes l_{2}^{1}(x, m)+\left(l_{2}^{0}(x, \cdot) * \xi\right) \otimes m$;

(ii) $\lceil\xi \otimes m, x\rceil=-\xi \otimes l_{2}^{1}(x, m)-\left(l_{2}^{0}(x, \cdot)^{*} \xi\right) \otimes m+\xi(x)\left(l_{1}(m)-l_{2}^{1}(m, \cdot)\right)$; 
(iii) $\lceil\xi \otimes m, \eta \otimes n\rceil=\left\langle l_{1}^{*} \eta, m\right\rangle \xi \otimes n-\left\langle l_{1}^{*} \xi, n\right\rangle \eta \otimes m$, equivalently, we have

$$
\lceil\phi, \psi\rceil=\phi \circ l_{1}^{*} \circ \psi-\psi \circ l_{1}^{*} \circ \phi .
$$

Proof. Using the property of the Dorfman bracket, it is straightforward to obtain (i) and (ii). We only give the proof of (iii). We have

$$
\begin{aligned}
\lceil\xi \otimes m, \eta \otimes n\rceil & =\eta \otimes \rho(\xi \otimes m)(n)+\lceil\xi \otimes m, \eta\rceil \otimes n \\
& =\rho(\xi)(n) \eta \otimes m-\lceil\eta, \xi \otimes m\rceil \otimes n \\
& =-l_{1}^{*} \xi(n) \eta \otimes m+l_{1}^{*} \eta(m) \xi \otimes n .
\end{aligned}
$$

Let it act on an arbitrary $\alpha \in \mathfrak{g}_{-1}^{*}$. We obtain

$$
\lceil\xi \otimes m, \eta \otimes n\rceil(\alpha)=\alpha(n) l_{1}^{*} \eta(m) \xi-\alpha(m) l_{1}^{*} \xi(n) \eta=\left(\phi \circ l_{1}^{*} \circ \psi-\psi \circ l_{1}^{*} \circ \phi\right)(\alpha) .
$$

This finishes the proof.

Remark 4.4. Note that the bracket (16) is naturally skew-symmetric and satisfies the Jacobi identity. Thus, it is a Lie bracket. It has already appeared in other places, e.g. see [37, Proposition 3.1] for more details.

Given a Courant algebroid $E \longrightarrow M$, using the skew-symmetric Courant bracket, we get a 2-term $L_{\infty^{-}}$ algebra structure on $C^{\infty}(M) \oplus \Gamma(E)$. Now consider the Courant algebroid (14) obtained from a 2-term $L_{\infty}$-algebra. Since it is linear, we pick linear functions on $\mathfrak{g}_{-1}^{*}$ as the degree -1 part and $\mathfrak{g}_{0} \oplus\left(\mathfrak{g}_{-1} \otimes \mathfrak{g}_{0}^{*}\right)$ as the degree 0 part. Before we give the precise structure of the corresponding 2 -term $L_{\infty}$-algebra, we give some properties of the skew-symmetric Courant bracket $\llbracket \cdot, \cdot \rrbracket$.

Corollary 4.5. With the above notations, for any $x, y, z \in \mathfrak{g}_{0}$, and $\xi \otimes m, \eta \otimes n \in \mathfrak{g}_{0}^{*} \otimes \mathfrak{g}_{-1}$, we have

$$
\left\{\begin{aligned}
\llbracket x, y \rrbracket & =x \circ y=l_{2}^{0}(x, y)+l_{3}(x, y, \cdot) ; \\
\llbracket x, \xi \otimes m \rrbracket & =\xi \otimes l_{2}^{1}(x, m)+\left(l_{2}^{0}(x, \cdot)^{*} \xi\right) \otimes m-\frac{1}{2} \xi(x)\left(l_{1}(m)-l_{2}^{1}(m, \cdot)\right) ; \\
\llbracket \xi \otimes m, \eta \otimes n \rrbracket & =l_{1}^{*} \eta(m) \xi \otimes n-l_{1}^{*} \xi(n) \eta \otimes m .
\end{aligned}\right.
$$

Now we can give the main theorem in this section.

Theorem 4.6. Given a 2 -term $L_{\infty}$-algebra $\mathfrak{g}=\left(\mathfrak{g}_{-1} \stackrel{l_{1}}{\longrightarrow} \mathfrak{g}_{0}, l_{2}=l_{2}^{0}+l_{2}^{1}, l_{3}\right)$, we can obtain a new 2-term $L_{\infty}$-algebra $\tilde{\mathfrak{g}}=\left(\mathfrak{g}_{-1} \stackrel{\tilde{l}_{1}}{\longrightarrow} \mathfrak{g}_{0} \oplus\left(\mathfrak{g}_{-1} \otimes \mathfrak{g}_{0}^{*}\right), \tilde{l}_{2}=\tilde{l}_{2}^{0}+\tilde{l}_{2}^{1}, \tilde{l}_{3}\right)$ from the corresponding Courant algebroid (14), in which $\tilde{l}_{1}=D$ (given by (15)), $\tilde{l}_{2}^{0}$ is given by (17)), $\tilde{l}_{2}^{1}$ and $\tilde{l}_{3}$ are given by

$$
\tilde{l}_{2}^{1}(x+\xi \otimes m, n)=\frac{1}{2} l_{2}^{1}(x, n)+\frac{1}{2}\left\langle\xi, l_{1}(n)\right\rangle m,
$$

and

$$
\begin{aligned}
& \tilde{l}_{3}\left(x_{1}+\xi_{1} \otimes m_{1}, x_{2}+\xi_{2} \otimes m_{2}, x_{3}+\xi_{3} \otimes m_{3}\right) \\
= & -\frac{1}{2} l_{3}\left(x_{1}, x_{2}, x_{3}\right)-\frac{1}{2}\left(\left\langle l_{2}^{0}\left(x_{1}, x_{2}\right), \xi_{3}\right\rangle m_{3}+c . p .\right) \\
& -\frac{1}{4}\left(\left\langle\xi_{1}, x_{2}\right\rangle\left\langle\xi_{3}, l_{1} m_{1}\right\rangle m_{3}-\left\langle\xi_{2}, x_{1}\right\rangle\left\langle\xi_{3}, l_{1} m_{2}\right\rangle m_{3}+\text { c.p. }\right) \\
& -\frac{1}{4}\left(\left\langle\xi_{2}, x_{3}\right\rangle l_{2}^{1}\left(x_{1}, m_{2}\right)-\left\langle\xi_{3}, x_{2}\right\rangle l_{2}^{1}\left(x_{1}, m_{3}\right)+\text { c.p. }\right) .
\end{aligned}
$$


Proof. It follows from Theorem [2.5, Proposition 4.2 and Corollary 4.5.

Now we give some examples to illustrate that the construction given in the above theorem is full of interest.

Example 4.7. (omni-Lie algebra) Let $V$ be a vector space. Consider the abelian 2-term $L_{\infty}$-algebra $\left(V \stackrel{\mathrm{id}}{\longrightarrow} V, l_{2}=0, l_{3}=0\right)$. By Theorem 4.6. we get a new 2 -term $L_{\infty}$-algebra $\left(V \stackrel{\dot{\mathrm{i}}}{\longrightarrow} V \oplus \mathfrak{g l}(V), \tilde{l}_{2}, \tilde{l}_{3}\right)$, in which $\dot{i}$ is the natural inclusion, and

$$
\left\{\begin{aligned}
\tilde{l}_{2}^{1}(u+A, m) & =\frac{1}{2} A m \\
\tilde{l}_{2}^{0}(u+A, v+B) & =\frac{1}{2}(A v-B u)+[A, B] \\
\tilde{l}_{3}(u+A, v+B, w+C) & =-\frac{1}{4}([A, B] w+[B, C] u+[C, A] v),
\end{aligned}\right.
$$

for all $u, v, w \in V_{0}=V, m \in V_{-1}=V$ and $A, B, C \in \mathfrak{g l}(V)$. This 2-term $L_{\infty}$-algebra is the one associated to the omni-Lie algebra $V \oplus \mathfrak{g l}(V)$. See [36, 41] for more details.

Example 4.8. (2-term $L_{\infty}$-algebra of string type) Let $\left(\mathfrak{k},[\cdot, \cdot]_{\mathfrak{k}}\right)$ be a Lie algebra. Consider the 2-term $L_{\infty}$-algebra $\left(\mathbb{R} \stackrel{0}{\longrightarrow} \mathfrak{k}, l_{2}=[\cdot, \cdot]_{\mathfrak{k}}, l_{3}=0\right)$. By Theorem 4.6, we get a new 2-term $L_{\infty}$-algebra $\left(\mathbb{R} \stackrel{0}{\longrightarrow} \mathfrak{k} \oplus \mathfrak{k}^{*}, \tilde{l}_{2}, \tilde{l}_{3}\right)$, where $\tilde{l}_{2}$ and $\tilde{l}_{3}$ are given by

$$
\left\{\begin{aligned}
\tilde{l}_{2}^{1}(u+\xi, r) & =0, \\
\tilde{l}_{2}^{0}(u+\xi, v+\eta) & =[u, v]_{\mathfrak{k}}+\operatorname{ad}_{u}^{*} \eta-\operatorname{ad}_{v}^{*} \xi \\
\tilde{l}_{3}(u+\xi, v+\eta, w+\zeta) & =-\frac{1}{2}\left(\left\langle[u, v]_{\mathfrak{k}}, \zeta\right\rangle+\left\langle[v, w]_{\mathfrak{k}}, \xi\right\rangle+\left\langle[w, u]_{\mathfrak{k}}, \eta\right\rangle\right),
\end{aligned}\right.
$$

for all $u, v, w \in \mathfrak{k}, \xi, \eta, \zeta \in \mathfrak{k}^{*}$ and $r \in \mathbb{R}$. This is exactly the 2-term $L_{\infty}$-algebra of string type studied in 36.

Example 4.9. Let $\left(\mathfrak{k},[\cdot, \cdot]_{\mathfrak{k}}, K\right)$ be a quadratic Lie algebra, and $\left(\mathbb{R} \stackrel{0}{\longrightarrow} \mathfrak{k}, l_{2}, l_{3}\right)$ the corresponding string Lie 2-algebra. More precisely,

$$
l_{2}^{0}(u, v)=[u, v]_{\mathfrak{k}}, l_{2}^{1}(u, r)=0, l_{3}(u, v, w)=K\left([u, v]_{\mathfrak{k}}, w\right), \forall u, v, w \in \mathfrak{k}, r \in \mathbb{R} .
$$

Then we get a new 2 -term $L_{\infty}$-algebra $\left(\mathbb{R} \stackrel{0}{\longrightarrow} \mathfrak{k} \oplus \mathfrak{k}^{*}, \tilde{l}_{2}, \tilde{l}_{3}\right)$, in which

$$
\left\{\begin{aligned}
\tilde{l}_{2}^{1}(u+\xi, r) & =0 \\
\tilde{l}_{2}^{0}(u+\xi, v+\eta) & =[u, v]_{\mathfrak{k}}+K\left([u, v]_{\mathfrak{k}}, \cdot\right)+\operatorname{ad}_{u}^{*} \eta-\operatorname{ad}_{v}^{*} \xi \\
\tilde{l}_{3}(u+\xi, v+\eta, w+\zeta) & =-\frac{1}{2} K\left([u, v]_{\mathfrak{k}}, w\right)-\frac{1}{2}\left(\left\langle[u, v]_{\mathfrak{k}}, \zeta\right\rangle+\left\langle[v, w]_{\mathfrak{k}}, \xi\right\rangle+\left\langle[w, u]_{\mathfrak{k}}, \eta\right\rangle\right),
\end{aligned}\right.
$$

for all $u, v, w \in \mathfrak{k}, r \in \mathbb{R}$ and $\xi, \eta, \zeta \in \mathfrak{k}^{*}$.

Example 4.10. Consider the 2 -term $L_{\infty}$-algebra $\left(\mathfrak{k} \stackrel{\mathrm{id}}{\longrightarrow} \mathfrak{k}, l_{2}=[\cdot, \cdot]_{\mathfrak{k}}, l_{3}=0\right)$, where $\left(\mathfrak{k},[\cdot, \cdot]_{\mathfrak{k}}\right)$ is a Lie algebra. By Theorem 4.6, we obtain a new 2-term $L_{\infty}$-algebra $\left(\mathfrak{k} \stackrel{\tilde{l}_{1}}{\longrightarrow} \mathfrak{k} \oplus \mathfrak{g l}(\mathfrak{k}), \tilde{l}_{2}, \tilde{l}_{3}\right)$, where

$$
\left\{\begin{aligned}
\tilde{l}_{1}(m) & =m-\operatorname{ad}_{m} \\
\tilde{l}_{2}^{1}(u+A, m) & =\frac{1}{2}[u, m]_{\mathfrak{k}}+\frac{1}{2} A m, \\
\tilde{l}_{2}^{0}(u+A, v+B) & =[u, v]_{\mathfrak{k}}+\frac{1}{2}(A v-B u)+\left[\operatorname{ad}_{u}, B\right]+\left[A, \operatorname{ad}_{v}\right]+\frac{1}{2}\left(\operatorname{ad}_{B u}-\operatorname{ad}_{A v}\right)+[A, B], \\
\tilde{l}_{3}(u+A, v+B, w+C) & =-\frac{1}{2} C[u, v]_{\mathfrak{k}}-\frac{1}{4}[A, B] w-\frac{1}{4}\left([u, B w]_{\mathfrak{k}}+[B u, w]_{\mathfrak{k}}\right)+c . p .
\end{aligned}\right.
$$

for all $u, v, w \in \mathfrak{k}, m \in \mathfrak{k}$ and $A, B, C \in \mathfrak{g l}(\mathfrak{k})$. This 2-term $L_{\infty}$-algebra can be viewed as a deformation of the one associated to the omni-Lie algebra. It can also be viewed as a linearization of the Courant algebroid $T M \oplus T_{\pi}^{*} M$, where $T_{\pi}^{*} M$ denotes the Lie algebroid associated to the Poisson manifold $(M, \pi)$. We will study its full properties in a separate paper. 
Now let us consider the relation between the 2 -term $L_{\infty}$-algebra given in the above theorem and the original 2-term $L_{\infty}$-algebra.

Theorem 4.11. With the above notations, there is an $L_{\infty}$-algebra homomorphism $F=\left(F_{0}=\mathrm{pr}_{1}, F_{1}=\right.$ $\left.\mathrm{id}, F_{2}\right)$ from $\tilde{\mathfrak{g}}$ to $\mathfrak{g}$, in which $\mathrm{pr}_{1}$ is the projection to the first component, and $F_{2}: \wedge^{2}\left(\mathfrak{g}_{0} \oplus\left(\mathfrak{g}_{0}^{*} \otimes \mathfrak{g}_{-1}\right)\right) \longrightarrow$ $\mathfrak{g}_{-1}$ is given by

$$
F_{2}(x+\xi \otimes m, y+\eta \otimes n)=\frac{1}{2}(\langle\xi, y\rangle m-\langle x, \eta\rangle n)=\frac{1}{2}(x+\xi \otimes m, y+\eta \otimes n)_{-},
$$

for all $x, y \in \mathfrak{g}_{0}, \quad \xi \otimes m, \eta \otimes n \in \mathfrak{g}_{0}^{*} \otimes \mathfrak{g}_{-1}$.

Proof. We verify the conditions of an $L_{\infty}$-algebra homomorphism step by step. It is obvious that

$$
F_{0} \circ D=l_{1} \circ F_{1} \text {. }
$$

Then, by Corollary 4.5, we obtain

$$
F_{0} \tilde{l}_{2}^{0}(x+\xi \otimes m, y+\eta \otimes n)=l_{2}^{0}(x, y)+\frac{1}{2}\left(\langle\xi, y\rangle l_{1} m-\langle x, \eta\rangle l_{1} n\right) .
$$

On the other hand, it is obvious that

$$
l_{2}^{0}\left(F_{0}(x+\xi \otimes m), F_{0}(y+\eta \otimes n)\right)=l_{2}^{0}(x, y) .
$$

Therefore, we have

$$
\begin{aligned}
& F_{0} \tilde{l}_{2}^{0}(x+\xi \otimes m, y+\eta \otimes n)-l_{2}^{0}\left(F_{0}(x+\xi \otimes m), F_{0}(y+\eta \otimes n)\right) \\
= & \frac{1}{2}\left(\langle\xi, y\rangle l_{1} m-\langle x, \eta\rangle l_{1} n\right) \\
= & l_{1} F_{2}(x+\xi \otimes m, y+\eta \otimes n) .
\end{aligned}
$$

By (18), we obtain

$$
F_{1} \tilde{l}_{2}^{1}(x+\xi \otimes m, n)=\frac{1}{2} l_{2}^{1}(x, n)+\frac{1}{2}\left\langle\xi, l_{1}(n)\right\rangle m,
$$

which implies that

$$
F_{1} \tilde{l}_{2}^{1}(x+\xi \otimes m, n)-l_{2}^{1}\left(F_{0}(x+\xi \otimes m), F_{1}(n)\right)=-\frac{1}{2} l_{2}^{1}(x, n)+\frac{1}{2}\left\langle\xi, l_{1}(n)\right\rangle m .
$$

On the other hand, we have

$$
F_{2}(x+\xi \otimes m, D n)=F_{2}\left(x+\xi \otimes m, l_{1}(n)-l_{2}^{1}(n, \cdot)\right)=-\frac{1}{2} l_{2}^{1}(x, n)+\frac{1}{2}\left\langle\xi, l_{1}(n)\right\rangle m .
$$

Thus, we have

$$
F_{1} \tilde{l}_{2}^{1}(x+\xi \otimes m, n)-l_{2}^{1}\left(F_{0}(x+\xi \otimes m), F_{1}(n)\right)=F_{2}(x+\xi \otimes m, D n) .
$$

It is left to prove the compatibility condition, that is, for all $a, b, c \in \mathfrak{g}_{0} \oplus \mathfrak{g}_{0}^{*} \otimes \mathfrak{g}_{-1}$,

$$
l_{2}^{1}\left(F_{0} a, F_{2}(b, c)\right)+c . p .+l_{3}\left(F_{0} a, F_{0} b, F_{0} c\right)=F_{2}\left(\tilde{l}_{2}^{0}(a, b), c\right)+c \cdot p .+F_{1} \tilde{l}_{3}(a, b, c) .
$$


First, for all $a, b, c \in \mathfrak{g}_{-1} \otimes \mathfrak{g}_{0}^{*}$, it is obvious that both sides of the equation are 0 . While for all $a, b, c \in \mathfrak{g}_{0}$ (we use the notation $x, y, z$ instead), it is obvious that the left hand side is equal to $l_{3}(x, y, z)$. It is not hard to see that

$$
\begin{aligned}
F_{2}\left(\tilde{l}_{2}^{0}(x, y), z\right)+c . p . & =F_{2}\left(l_{3}(x, y, \cdot), z\right)+c \cdot p \cdot=\frac{1}{2} l_{3}(x, y, z)+c \cdot p \cdot=\frac{3}{2} l_{3}(x, y, z) \\
F_{1} \tilde{l}_{3}(x, y, z) & ==-\frac{1}{2} l_{3}(x, y, z), \quad \text { by Theorem 4.6 }
\end{aligned}
$$

which implies that (24) holds for all $x, y, z \in \mathfrak{g}_{0}$. For $x, y$ in $\mathfrak{g}_{0}$ and $\xi \otimes m$ in $\mathfrak{g}_{0}^{*} \otimes \mathfrak{g}_{-1}$, we have

$$
\begin{aligned}
l_{2}^{1}\left(F_{0} x, F_{2}(y, \xi \otimes m)\right)+c . p .+l_{3}\left(F_{0} x, F_{0} y, F_{0}(\xi \otimes m)\right) & =l_{2}^{1}\left(x,-\frac{1}{2}\langle\xi, y\rangle m\right)+l_{2}^{1}\left(y, \frac{1}{2}\langle\xi, x\rangle m\right) \\
& =-\frac{1}{2}\langle y, \xi\rangle l_{2}^{1}(x, m)+\frac{1}{2}\langle x, \xi\rangle l_{2}^{1}(y, m),
\end{aligned}
$$

and

$$
\begin{aligned}
& F_{2}\left(\tilde{l}_{2}^{0}(x, y), \xi \otimes m\right)+c . p .+F_{1} \tilde{l}_{3}(x, y, \xi \otimes m) \\
= & \left(-\frac{1}{2}-\frac{1}{6}\right)\langle\llbracket x, y \rrbracket, \xi\rangle m+\left(\frac{1}{2}-\frac{1}{6}\right)\langle\llbracket y, \xi \otimes m \rrbracket, x\rangle+\left(\frac{1}{2}-\frac{1}{6}\right)\langle\llbracket \xi \otimes m, x \rrbracket, y\rangle \\
= & -\frac{2}{3}\left\langle l_{2}^{0}(x, y), \xi\right\rangle m+\frac{1}{3}\left\{\langle\xi, x\rangle l_{2}^{1}(y, m)-\left\langle\xi, l_{2}^{0}(y, x)\right\rangle m+\frac{1}{2}\langle\xi, y\rangle l_{2}^{1}(m, x)\right\} \\
& +\frac{1}{3}\left\{-\langle\xi, y\rangle l_{2}^{1}(x, m)+\left\langle\xi, l_{2}^{0}(x, y)\right\rangle m-\frac{1}{2}\langle\xi, x\rangle l_{2}^{1}(m, y)\right\} \\
= & -\frac{1}{2}\langle y, \xi\rangle l_{2}^{1}(x, m)+\frac{1}{2}\langle x, \xi\rangle l_{2}^{1}(y, m),
\end{aligned}
$$

which implies that (24) holds for two elements in $\mathfrak{g}_{0}$ and one element in $\mathfrak{g}_{0}^{*} \otimes \mathfrak{g}_{-1}$. At last, for $x \in \mathfrak{g}_{0}$ and $\xi \otimes m, \eta \otimes n \in \mathfrak{g}_{0}^{*} \otimes \mathfrak{g}_{-1}$, it is obvious that the left hand side of (24) is equal to 0 . Furthermore, we have

$$
\begin{aligned}
& F_{2}\left(\tilde{l}_{2}^{0}(x, \xi \otimes m), \eta \otimes n\right)+c . p .+F_{1} \tilde{l}_{3}(x, \xi \otimes m, \eta \otimes n) \\
= & \left(-\frac{1}{2}-\frac{1}{6}\right)\langle\llbracket x, \xi \otimes m \rrbracket, \eta\rangle n+\left(\frac{1}{2}-\frac{1}{6}\right)\langle\llbracket \xi \otimes m, \eta \otimes n \rrbracket, x\rangle+\left(-\frac{1}{2}-\frac{1}{6}\right)\langle\llbracket \eta \otimes n, x \rrbracket, \xi\rangle m \\
= & -\frac{2}{3} \times\left(-\frac{1}{2}\right)\langle\xi, x\rangle\left\langle l_{1} m, \eta\right\rangle n+\frac{1}{3}\left\{-\left\langle l_{1} m, \eta\right\rangle\langle\xi, x\rangle n+\left\langle l_{1} n, \xi\right\rangle\langle\eta, x\rangle m\right\} \\
& -\frac{2}{3} \times \frac{1}{2}\left\langle l_{1} n, \xi\right\rangle\langle\eta, x\rangle m \\
= & 0 .
\end{aligned}
$$

Thus, (24) holds for all $a, b, c \in \mathfrak{g}_{0} \oplus \mathfrak{g}_{0}^{*} \otimes \mathfrak{g}_{-1}$. By (21)-(24), we deduce that $F$ is an $L_{\infty}$-algebra homomorphism.

\section{Lie 2-algebras and quasi-Poisson groupoids}

In this section, we show that the Courant algebroid associated to a 2 -term $L_{\infty}$-algebra is the double of a Lie quasi-bialgebroid. Then, by integration, we obtain a quasi-Poisson groupoid. Since a 2 -term $L_{\infty^{-}}$ algebra is equivalent to a Lie 2-algebra, this quasi-Poisson groupoid can serve as the geometric structure on the dual of a Lie 2-algebra. 
Let $\mathfrak{g}=\left(\mathfrak{g}_{-1} \stackrel{l_{1}}{\longrightarrow} \mathfrak{g}_{0}, l_{2}=l_{2}^{0}+l_{2}^{1}, l_{3}\right)$ be a 2 -term $L_{\infty}$-algebra. The vector bundle $E \longrightarrow M$ given by (14) can be decomposed as $A \oplus A^{*}$, where $A=\mathfrak{g}_{-1}^{*} \times \mathfrak{g}_{0}^{*} \longrightarrow \mathfrak{g}_{-1}^{*}$ is a trivial vector bundle. By Lemma 2.3 $(k=-1)$, the 2-term $L_{\infty}$-algebra structure $l=l_{1}+\left(l_{2}^{1}+l_{2}^{0}\right)+l_{3}$, where $l_{1} \in \mathfrak{g}_{-1}^{*} \otimes \mathfrak{g}_{0}, l_{2}^{0} \in \wedge^{2} \mathfrak{g}_{0}^{*} \otimes \mathfrak{g}_{0}, l_{2}^{1} \in$ $\mathfrak{g}_{0}^{*} \wedge \mathfrak{g}_{-1}^{*} \otimes \mathfrak{g}_{-1}, l_{3} \in \wedge^{3} \mathfrak{g}_{0}^{*} \otimes \mathfrak{g}_{-1}$, satisfies $\{l, l\}=0$. By the degree reason, $\{l, l\}=0$ implies that

$$
\left\{l_{1}, l_{1}\right\}=0 \quad\left\{l_{1}, l_{2}\right\}=0, \quad \frac{1}{2}\left\{l_{2}, l_{2}\right\}+\left\{l_{1}, l_{3}\right\}=0, \quad\left\{l_{2}, l_{3}\right\}=0 .
$$

Comparing this with (9), we can see that Eq. (25) implies Eq. (9) for $\mu=-l_{1}, \gamma=-l_{2}=-l_{2}^{0}-l_{2}^{1}, \phi=$ $-l_{3}$. Therefore, we have

Theorem 5.1. Let $\mathfrak{g}=\left(\mathfrak{g}_{-1} \stackrel{l_{1}}{\longrightarrow} \mathfrak{g}_{0}, l_{2}=l_{2}^{0}+l_{2}^{1}, l_{3}\right)$ be a 2-term $L_{\infty}$-algebra. Then $(A,-l)$ is a Lie quasi-bialgebroid, where $A=\mathfrak{g}_{-1}^{*} \times \mathfrak{g}_{0}^{*} \longrightarrow \mathfrak{g}_{-1}^{*}$ and $l=l_{1}+\left(l_{2}^{1}+l_{2}^{0}\right)+l_{3}$, and the Courant algebroid $E$ is the double of the Lie quasi-bialgebroid $(A,-l)$.

Now we give the precise structure on $A$ and $A^{*}$ using the general method of differential geometry. By Theorem 5.1 and Proposition 4.2] we have

Corollary 5.2. The Lie algebroid structure on $A$, determined by $-l_{1}$, is given by

(i) for any constant section $\xi \in \mathfrak{g}_{0}^{*}$, the anchor $\rho_{A}$ is given by $\rho_{A}(\xi)=-l_{1}^{*}(\xi)$;

(ii) for any constant sections $\xi, \eta \in \mathfrak{g}_{0}^{*}$, we have $[\xi, \eta]_{A}=0$;

(iii) for any constant section $\xi \in \mathfrak{g}_{0}^{*}$ and linear section $\eta \otimes n \in \mathfrak{g}_{0}^{*} \otimes \mathfrak{g}_{-1}$, we have

$$
[\xi, \eta \otimes n]_{A}=\left\langle\xi, l_{1}(n)\right\rangle \eta
$$

(iv) for any linear sections $\xi \otimes m, \eta \otimes n \in \mathfrak{g}_{0}^{*} \otimes \mathfrak{g}_{-1}$, we have

$$
[\xi \otimes m, \eta \otimes n]_{A}=\left\langle l_{1}^{*} \eta, m\right\rangle \xi \otimes n-\left\langle l_{1}^{*} \xi, n\right\rangle \eta \otimes m .
$$

Thus, $A$ is an action Lie algebroid of the abelian Lie algebra $\mathfrak{g}_{0}^{*}$ acting on $\mathfrak{g}_{-1}^{*}$ via $-l_{1}^{*}$, which sends an element $\xi \in \mathfrak{g}_{0}^{*}$ to a constant vector field $-l_{1}^{*}(\xi) \in \mathfrak{g}_{-1}^{*}$. The corresponding Chevalley-Eilenberg coboundary operator $d_{A}: \Gamma\left(\wedge^{\bullet} A^{*}\right) \longrightarrow \Gamma\left(\wedge^{\bullet+1} A^{*}\right)$ is determined by

$$
d_{A} m=\left\{-l_{1}, m\right\}=l_{1}(m) .
$$

Corollary 5.3. For all constant section $x \in \mathfrak{g}_{0}$ of $A^{*},-l_{2}^{1}$ gives rise to the anchor map $\rho_{A^{*}}$ of $A^{*}$ via

$$
\rho_{A^{*}}(x)=l_{2}^{1}(x, \cdot),
$$

which is a linear vector field. For all constant sections $x, y \in \mathfrak{g}_{0},-l_{2}^{0}$ gives rise to the bracket operation on $A^{*}$ :

$$
[x, y]_{A^{*}}=l_{2}^{0}(x, y) \text {. }
$$

The Jacobi identity of $[\cdot, \cdot]_{A^{*}}$ is controlled by $\phi=-l_{3} \in \wedge^{3} \mathfrak{g}_{0}^{*} \otimes \mathfrak{g}_{-1} \subset \Gamma\left(\wedge^{3} A\right)$. More precisely, we have

$$
\left[[x, y]_{A^{*}}, z\right]_{A^{*}}+\text { c.p. }=d_{A} \phi(x, y, z)+\phi\left(d_{A} x, y, z\right)-\phi\left(x, d_{A} y, z\right)+\phi\left(x, y, d_{A} z\right) .
$$

Now we can restate Theorem 5.1 using usual language of differential geometry. This is necessary since we will consider the integration of Lie quasi-bialgebroids at the end of this section. 
Corollary 5.4. Given a 2-term $L_{\infty}$-algebra $\mathfrak{g}=\left(\mathfrak{g}_{-1} \stackrel{l_{1}}{\longrightarrow} \mathfrak{g}_{0}, l_{2}=l_{2}^{0}+l_{2}^{1}, l_{3}\right)$, we obtain a Lie quasibialgebroid $(A, \delta, \phi)$, where the Lie algebroid $A=\mathfrak{g}_{-1}^{*} \times \mathfrak{g}_{0}^{*} \longrightarrow \mathfrak{g}_{-1}^{*}$ is given by Corollary 5.2. $\delta$ : $\Gamma\left(\wedge^{k} A\right) \longrightarrow \Gamma\left(\wedge^{k+1} A\right)$ is the generalized Chevalley-Eilenberg operator determined by the anchor $\rho_{A^{*}}$ and the bracket $[\cdot, \cdot]_{A^{*}}$ given in Corollary [5.3, and $\phi=-l_{3}$.

In the last section, we have seen that, given a 2 -term $L_{\infty}$-algebra, there is a new 2 -term $L_{\infty}$-algebra associated to the Courant algebroid $E$ (Theorem 4.6), and there is an $L_{\infty}$-algebra homomorphism from the new 2-term $L_{\infty}$-algebra to the original one (Theorem 4.11). In this section, we see that the Courant algebroid is exactly the double of the Lie quasi-bialgebroid $(A, \delta, \phi)$. In fact, we can obtain a more general result than Theorem 4.11. Namely, given any Lie quasi-bialgebroid $(A, \delta, \phi)$, we can obtain a 2 -term $L_{\infty^{-}}$ algebra $C^{\infty}(M) \oplus \operatorname{ker}\left(\left.d_{A}\right|_{\Gamma\left(A^{*}\right)}\right)$, where $d_{A}$ is the coboundary operator associated to Lie algebroid $A$ and $\left.d_{A}\right|_{\Gamma\left(A^{*}\right)}: \Gamma\left(A^{*}\right) \longrightarrow \Gamma\left(\wedge^{2} A^{*}\right)$ is the restriction of $d_{A}$ on $\Gamma\left(A^{*}\right)$. Furthermore, we construct an $L_{\infty}$-algebra homomorphism from the 2 -term $L_{\infty}$-algebra associated the Courant algebroid $A \oplus A^{*}$ to $C^{\infty}(M) \oplus \operatorname{ker}\left(\left.d_{A}\right|_{\Gamma\left(A^{*}\right)}\right)$.

Theorem 5.5. Let $(A, \delta, \phi)$ be a Lie quasi-bialgebroid. Then there is a 2-term $L_{\infty}$-algebra structure on $C^{\infty}(M) \oplus \operatorname{ker}\left(\left.d_{A}\right|_{\Gamma\left(A^{*}\right)}\right)$, where $C^{\infty}(M)$ is of degree $-1, \operatorname{ker}\left(\left.d_{A}\right|_{\Gamma\left(A^{*}\right)}\right)$ is of degree 0 , and $\left\{l_{i}\right\}$ are given by

$$
\left\{\begin{aligned}
l_{1}(f) & =d_{A} f, & & \forall f \in C^{\infty}(M), \\
l_{2}\left(e_{1}, e_{2}\right) & =\left[e_{1}, e_{2}\right]_{A^{*}} & & \forall e_{1}, e_{2} \in \operatorname{ker}\left(\left.d_{A}\right|_{\Gamma\left(A^{*}\right)}\right), \\
l_{2}\left(e_{1}, f\right) & =\rho_{A^{*}}\left(e_{1}\right)(f) & & \forall e_{1} \in \operatorname{ker}\left(\left.d_{A}\right|_{\Gamma\left(A^{*}\right)}\right), f \in C^{\infty}(M), \\
l_{3}\left(e_{1}, e_{2}, e_{3}\right) & =-\phi\left(e_{1}, e_{2}, e_{3}\right) & & \forall e_{1}, e_{2}, e_{3} \in \operatorname{ker}\left(\left.d_{A}\right|_{\Gamma\left(A^{*}\right)}\right) .
\end{aligned}\right.
$$

Furthermore, there is an $L_{\infty}$-algebra homomorphism $F=\left(F_{0}=\mathrm{pr}_{2}, F_{1}=\mathrm{id}, F_{2}\right)$ from the 2-term $L_{\infty}$-algebra associated to the Courant algebroid $A \oplus A^{*}, C^{\infty}(M) \oplus\left(\Gamma(A) \oplus \operatorname{ker}\left(\left.d_{A}\right|_{\Gamma\left(A^{*}\right)}\right)\right)$, to $C^{\infty}(M) \oplus$ $\operatorname{ker}\left(\left.d_{A}\right|_{\Gamma\left(A^{*}\right)}\right)$, where $\operatorname{pr}_{2}$ is the projection to the second component, and $F_{2}$ is given by

$$
F_{2}\left(e_{1}, e_{2}\right)=\frac{1}{2}\left(e_{1}, e_{2}\right)_{-}, \quad \forall e_{1}, e_{2} \in \Gamma(A) \oplus \operatorname{ker}\left(\left.d_{A}\right|_{\Gamma\left(A^{*}\right)}\right) .
$$

Proof. Since $A$ is a Lie algebroid, we have $d_{A}^{2}=0$, i.e. $\operatorname{Im}\left(d_{A}\right) \subset \operatorname{ker}\left(d_{A}\right)$. Thus, $l_{1}$ is well-defined. By the fact that $(A, \delta, \phi)$ is a Lie quasi-bialgebroid, it is straightforward to see that $\left\{l_{i}\right\}$ is a 2-term $L_{\infty}$-algebra structure. Furthermore, it is not hard to see that the Courant bracket on $\Gamma(A) \oplus \operatorname{ker}\left(\left.d_{A}\right|_{\Gamma\left(A^{*}\right)}\right)$ is closed, which implies that the 2-term $L_{\infty}$-algebra $C^{\infty}(M) \oplus\left(\Gamma(A) \oplus \Gamma\left(A^{*}\right)\right)$ associated to the Courant algebroid $A \oplus A^{*}$ can be reduced to $C^{\infty}(M) \oplus\left(\Gamma(A) \oplus \operatorname{ker}\left(\left.d_{A}\right|_{\Gamma\left(A^{*}\right)}\right)\right)$. We can also prove that $F$ is indeed a homomorphism similar as the proof of Theorem 4.11.

Next, we return to the Lie quasi-bialgebroid $(A, \delta, \phi)$ associated to a 2 -term $L_{\infty}$-algebra. Clearly, the Lie algebroid $A$ can be integrated to an action groupoid $\Gamma: \mathfrak{g}_{-1}^{*} \times \mathfrak{g}_{0}^{*} \rightrightarrows \mathfrak{g}_{-1}^{*}$ with the abelian group structure on $\mathfrak{g}_{0}^{*}$, where the source, target and inclusion maps are given by

$$
s(\alpha, \xi)=\alpha, t(\alpha, \xi)=\alpha+l_{1}^{*} \xi, i(\alpha)=(\alpha, 0),
$$

for all $(\alpha, \xi) \in \mathfrak{g}_{-1}^{*} \times \mathfrak{g}_{0}^{*}$. By Theorem 4.9 in [15], we know that $\Gamma$ has a quasi-Poisson structure, such that its corresponding Lie quasi-bialgebroid is exactly $(A, \delta, \phi)$. Recall that a quasi-Poisson groupoid is a triple $(\Gamma, \Pi, \phi)$, where $\Gamma$ is a Lie groupoid whose Lie algebroid is $A, \Pi \in \wedge^{2} \mathfrak{X}(\Gamma)$, and $\phi \in \Gamma\left(\wedge^{3} A\right)$, satisfying $\frac{1}{2}[\Pi, \Pi]_{S}=\overleftarrow{\phi}-\vec{\phi},[\Pi, \overleftarrow{\phi}]=0$. Generally, it is a rather difficult work to get the bivector field from the data of an ordinary Lie quasi-bialgebroid. Nevertheless, we can elaborate the quasi-Poisson structure in our specific case, since all the structures are determined by the information of the 2-term $L_{\infty}$-algebra. 
Theorem 5.6. The quasi-Poisson groupoid corresponding to $(A, \delta, \phi)$ is $\left(\Gamma, \Pi, \phi=-l_{3}\right)$, where $\Pi$ is characterized by

$$
\Pi(d x, d y)=-l_{2}^{0}(x, y), \quad \Pi(d x, d m)=-l_{2}^{1}(x, m), \quad \Pi(d m, d n)=-l_{2}^{1}\left(l_{1}(m), n\right),
$$

where $d$ is the usual de Rham differential, and $x, y \in \mathfrak{g}_{0}, m, n \in \mathfrak{g}_{-1}$ are linear functions on $\mathfrak{g}_{-1}^{*} \times \mathfrak{g}_{0}^{*}$.

We need to make some preparations to prove the theorem. Let us take a general action groupoid into account.

Let $M \triangleleft G$ be an action groupoid and $M \triangleleft \mathfrak{g}$ the corresponding Lie algebroid, where $\mathfrak{g}$ is the Lie algebra of Lie group $G$, and the anchor is given by the infinitesimal action of $\mathfrak{g}$ on $M$, i.e. a Lie algebra homomorphism $\widehat{\cdot}: \mathfrak{g} \longrightarrow \mathfrak{X}(M)$,

$$
\widehat{X}(x)=\frac{d}{d t} x \cdot \exp (-t X), \quad \forall X \in \mathfrak{g}, x \in M .
$$

We use $l_{a}\left(\right.$ resp. $\left.r_{a}\right)$ to denote the left(right)-translation on the Lie group $G$ and $l_{(x, a)}\left(r e s p\right.$. $\left.r_{(x, a)}\right)$ the left(right)-translation on the groupoid $M \triangleleft G$ respectively. Let $\left(l_{a}\right)_{*},\left(r_{a}\right)_{*}$ and $\left(l_{(x, a)}\right)_{*},\left(r_{(x, a)}\right)_{*}$ be the corresponding tangent maps.

For the Lie algebroid $A$ of a Lie groupoid $\Gamma$, when we say the left-translation $\overleftarrow{\Lambda}$ (right-translation $\vec{\Lambda}$ ) of $\Lambda \in \Gamma(A)$, we are identifying $\Gamma(A)$ with left-invariant (right-invariant) vector fields on $\Gamma$. We assume that the derivatives are all taken at $t=0$.

Lemma 5.7. Let $M \triangleleft G$ be an action Lie groupoid and $M \triangleleft \mathfrak{g}$ the corresponding Lie algebroid. For all $\phi \in \Gamma(M \triangleleft \mathfrak{g}),(x, a) \in M \times G$, we have

$$
\overleftarrow{\phi}_{(x, a)}=\left(0_{x},\left(l_{a}\right)_{* e} \phi_{x \cdot a}\right), \quad \vec{\phi}_{(x, a)}=\left(\left(\widehat{\phi_{x}}\right)_{x},\left(r_{a}\right)_{* e} \phi_{x}\right)
$$

Proof. For this action groupoid, the $s$-fiber over $x$ is $s^{-1}(x)=\{(x, a) ; \forall a \in G\}=\{x\} \times G$, and the $t$-fiber over $x$ is $t^{-1}(x)=\left\{\left(x \cdot a^{-1}, a\right) ; \forall a \in G\right\}$. The tangent map of $l_{(x, a)}: s^{-1}(x \cdot a) \longrightarrow s^{-1}(x)$ is $\left(l_{(x, a)}\right)_{*(x \cdot a, e)}: T_{(x \cdot a, e)} s^{-1}(x \cdot a) \longrightarrow T_{(x, a)} s^{-1}(x)$. Let $\phi: M \longrightarrow \mathfrak{g}$ be a section of $M \triangleleft \mathfrak{g}$ and $\gamma(t)$ a smooth curve on $G$ satisfying $\gamma(0)=e, \gamma^{\prime}(0)=\phi(x \cdot a)$. Then we have

$$
\overleftarrow{\phi}_{(x, a)}=\left(l_{(x, a)}\right)_{*} \phi_{x \cdot a}=\frac{d}{d t}(x, a)(x \cdot a, \gamma(t))=\frac{d}{d t}(x, a \gamma(t))=\left(0_{x},\left(l_{a}\right)_{* e} \phi_{x \cdot a}\right)
$$

Similarly, the tangent map of $r_{(x, a)}: t^{-1}(x) \longrightarrow t^{-1}(x \cdot a)$ is $\left(r_{(x, a)}\right)_{*(x, e)}: T_{(x, e)} t^{-1}(x) \longrightarrow T_{(x, a)} t^{-1}(x \cdot a)$. Let $\gamma(t)$ be a smooth curve on $G$ satisfying $\gamma(0)=e, \gamma^{\prime}(0)=\phi(x)$. When $\phi$ is considered as a rightinvariant vector field, we have

$$
\phi_{x}=\frac{d}{d t}(x, \gamma(-t))^{-1}=\frac{d}{d t}\left(x \cdot \gamma(-t), \gamma(-t)^{-1}\right) .
$$

Thus, we get

$$
\begin{aligned}
\vec{\phi}_{(x, a)} & =\left(r_{(x, a)}\right)_{*} \phi_{x}=\frac{d}{d t}\left(x \cdot \gamma(-t), \gamma(-t)^{-1}\right)(x, a) \\
& =\frac{d}{d t}(x \cdot \gamma(-t), \gamma(t) a)=\left(\left(\widehat{\phi_{x}}\right)_{x},\left(r_{a}\right)_{* e} \phi_{x}\right) .
\end{aligned}
$$

Proof of Theorem 5.6: According to Theorem 2.34 and Theorem 4.9 in [15, we only need to prove $\delta_{\Pi}=\delta$, that is, for any function $f \in C^{\infty}\left(\mathfrak{g}_{-1}^{*}\right)$ and any section $e \in \Gamma(A)$, we have $\overleftarrow{\delta f}=\left[t^{*} f, \Pi\right]_{S}, \overleftarrow{\delta e}=$ $[\overleftarrow{e}, \Pi]_{S}$. We only give the proof for a linear function $m \in \mathfrak{g}_{-1}$ and a constant section $\xi \in \mathfrak{g}_{0}^{*}$. 
By calculation, we have $t^{*}(m)=m-l_{1}(m)$. Considering the linear action of the abelian group $\mathfrak{g}_{0}^{*}$ on $\mathfrak{g}_{-1}^{*}$ and by Lemma 5.7, we have,

$$
\begin{aligned}
\overleftarrow{\delta m} & =\delta m-l_{1}(\delta m)=-l_{2}^{1}(m, \cdot)+l_{1}\left(l_{2}^{1}(m, \cdot)\right) \\
\overleftarrow{\delta \xi} & =\delta \xi-l_{1}(\delta \xi)=\delta \xi \\
\overleftarrow{\xi} & =\xi
\end{aligned}
$$

Thus, for a linear function $n+x$ on $\mathfrak{g}_{-1}^{*} \times \mathfrak{g}_{0}^{*}$, we have

$$
\overleftarrow{\delta m}(n+x)=-l_{2}^{1}(m, x)+l_{1}\left(l_{2}^{1}(m, x)\right)
$$

while

$$
\begin{aligned}
{\left[t^{*} m, \Pi\right]_{S}(n+x) } & =\Pi(d m, d n)+\Pi(d m, d x)-\Pi\left(d l_{1}(m), d n\right)-\Pi\left(d l_{1}(m), d x\right) \\
& =-l_{2}^{1}(m, x)+l_{2}^{0}\left(l_{1}(m), x\right) .
\end{aligned}
$$

Then, we have $\overleftarrow{\delta m}=\left[t^{*} m, \Pi\right]_{S}$. To prove $\delta \xi=[\xi, \Pi]_{S}$ as bivector fields, we just need to verify that it holds on two 1-forms on $\mathfrak{g}_{0}^{*}$, since it vanishes for all the other cases. By straightforward calculation, we have $\delta \xi(d x, d y)=-\left\langle\xi,[x, y]_{A^{*}}\right\rangle=-\left\langle\xi, l_{2}^{0}(x, y)\right\rangle$, and

$$
\begin{aligned}
{[\xi, \Pi]_{S}(d x, d y) } & =L_{\xi} \Pi(d x, d y) \\
& =-\left\langle\xi, l_{2}^{0}(x, y)\right\rangle-\Pi\left(L_{\xi} d x, d y\right)-\Pi\left(d x, L_{\xi} d y\right) \\
& =-\left\langle\xi, l_{2}^{0}(x, y)\right\rangle .
\end{aligned}
$$

Therefore, we complete the proof.

There is a one-to-one correspondence between 2-term chain complex $V_{1} \stackrel{\mathrm{d}}{\longrightarrow} V_{0}$ and 2-vector space $\mathbb{V}: V_{1} \oplus V_{0} \rightrightarrows V_{0}$. Actually, the 2 -vector space $\mathbb{V}$ is an action Lie groupoid $V_{0} \triangleleft V_{1}$, where $V_{1}$ is seen as an abelian group and the action is given by $v_{0} \cdot v_{1}:=v_{0}+\mathrm{d} v_{1}$, for all $v_{0} \in V_{0}, v_{1} \in V_{1}$.

Definition 5.8. Let $\mathbb{V}$ be a 2 -vector space. If $(\mathbb{V}, \Pi, \phi)$ is a quasi-Poisson groupoid such that the bivector field $\Pi$ and the trisection $\phi$ are both linear, we call $(\mathbb{V}, \Pi, \phi)$ a Lie-quasi-Poisson groupoid.

Consider the 2-vector space $\mathfrak{g}_{-1}^{*} \oplus \mathfrak{g}_{0}^{*} \rightrightarrows \mathfrak{g}_{-1}^{*}$ given by the 2-term chain complex $\mathfrak{g}_{0}^{*} \stackrel{l_{1}^{*}}{\longrightarrow} \mathfrak{g}_{-1}^{*}$, which is the dual of the complex $\mathfrak{g}_{-1} \stackrel{l_{1}}{\longrightarrow} \mathfrak{g}_{0}$. Consequently, by Theorem [5.6, we generalize the fact that there is a Lie-Poisson structure on the dual space of a Lie algebra to the case of Lie 2-algebras.

Theorem 5.9. The dual of a Lie 2-algebra is a Lie-quasi-Poisson groupoid.

We obtain our result through the integration of the Lie quasi-bialgebroid associated to a Lie 2-algebra. One can also obtain a quasi-Poisson Lie 2-group from a Lie 2-algebra via the integration of Lie 2-bialgebras. See [12] for more details.

\section{Acknowledgement}

We give our warmest thanks to Jim Stasheff and the referee for very helpful comments that improve the paper. We also give our special thanks to Noriaki Ikeda, Zhangju Liu, Pavol Ševera and Chenchang Zhu for very useful comments and discussions. We thank Marco Zambon for pointing out the reference [26] to us. 


\section{References}

[1] Alekseev, A. and Kosmann-Schwarzbach, Y.: Manin pairs and moment maps. J. Diff. Geom. 56 (2000), $133-165$.

[2] Alekseev, A., Kosmann-Schwarzbach, Y. and Meinrenken, E.: Quasi-Poisson manifolds. Canadian J. Math. 54 (2002), 3-29.

[3] Alexandrov, M., Kontsevich, M., Schwartz, A. and Zaboronsky, O.: The geometry of the master equation and topological quantum field theory. Int. J. Mod. Phys. A 12 (1997), 1405.

[4] Baez, J. C. and Crans, A. S.: Higher-Dimensional Algebra VI: Lie 2-Algebras. Theory and Appl. Categ. 12 (2004), 492-528.

[5] Baez, J. C., Hoffnung, A. E. and Rogers, C. L.: Categorified symplectic geometry and the classical string. Comm. Math. Phys., 293 (2010), no. 3, 701-725.

[6] Baez, J. C. and Rogers, C. L.: Categorified symplectic geometry and the string Lie 2-algebra. Homology, Homotopy Appl., 12 (2010), no. 1, 221-236.

[7] Bonavolontà, G. and Poncin, N.: On the category of Lie n-algebroids. J. Geom. Phys. 73 (2013), 79-90.

[8] Bruce, A.: From $L_{\infty}$-algebroids to higher Schouten/Poisson structures. Rep. Math. Phys. 67 (2011), no. 2, $157-177$.

[9] Cattaneo, A. S., Fiorenza, D. and Longoni, R.: Graded Poisson Algebras. Encyclopedia of Math. Phys., 2 (2006), 560-567.

[10] Cattaneo, A. S. and Felder, G.: Relative formality theorem and quantisation of coisotropic submanifolds. Adv. Math. 208 (2007), no. 2, 521-548.

[11] Cattaneo, A. S. and Zambon, M.: A supergeometric approach to Poisson reduction. Comm. Math. Phys. 318 (2013), no. 3, 675-716.

[12] Chen, Z., Stiénon, M. and Xu, P.: Poisson 2-groups. J. Diff. Geom. 94 (2013), no. 2, $209-240$.

[13] Fregier, Y. and Zambon, M.: Simultaneous deformations and Poisson geometry. Compos. Math. 151 (2015), no. 9, 1763-1790.

[14] Hansen, M. and Strobl, T.: First Class Constrained Systems and Twisting of Courant Algebroids by a Closed 4-form Fundamental interactions, 115-144, World Sci. Publ., Hackensack, NJ, 2010.

[15] Iglesias Ponte, D., Laurent-Gengoux, C. and Xu, P.: Universal lifting theorem and quasi-Poisson groupoids. J. Eur. Math. Soc. (JEMS) 14 (2012), no. 3, 681-731.

[16] Ikeda, N. and Xu, X.: Canonical functions, differential graded symplectic pairs in supergeometry, and AlexandrovKontsevich-Schwartz-Zaboronsky sigma models with boundaries. J. Math. Phys, 55 (2014), 113505.

[17] Khudaverdian, H. M. and Voronov, Th.: Higher Poisson brackets and differential forms. Geometric methods in physics, 203-215, AIP Conf. Proc., 1079, Amer. Inst. Phys., Melville, NY, 2008.

[18] Kosmann-Schwarzbach, Y.: Jacobian quasi-bialgebras and quasi-Poisson Lie groups. Mathematical aspects of classical field theory (Seattle, WA, 1991), 459-489, Contemp. Math., 132, Amer. Math. Soc., Providence, RI, 1992.

[19] Kosmann-Schwarzbach, Y.: Quasi, twisted, and all that... in Poisson geometry and Lie algebroid theory. The breadth of symplectic and Poisson geometry, 363-389, Progr. Math., 232, Birkhäuser Boston, Boston, MA, 2005.

[20] Kosmann-Schwarzbach, Y.: Poisson and symplectic functions in Lie algebroid theory. Higher structures in geometry and physics, 243-268, Progr. Math., 287, Birkhäuser/Springer, New York, 2011.

[21] Lada, T. and Markl, M.: Strongly homotopy Lie algebras. Comm. Algebra 23 (1995), no. 6, $2147-2161$.

[22] Lada, T. and Stasheff, J.: Introduction to sh Lie algebras for physicists. Int. Jour. Theor. Phys. 32 (1993), no. 7, 1087-1103.

[23] Liu, Z., Weinstein, A. and Xu, P.: Manin triples for Lie bialgebroids. J. Diff. Geom. 45 (1997), $547-574$.

24] Mackenzie, K. C. H.: Ehresmann doubles and Drinfel'd doubles for Lie algebroids and Lie bialgebroids. J. Reine Angew. Math. 658 (2011), 193-245.

[25] Mackenzie, K. C. H. and Xu, P.: Lie bialgebroids and Poisson groupoids. Duke Math. J. 73 (1994), no. 2, 415-452.

[26] Mehta, R. A.: On homotopy Poisson actions and reduction of symplectic Q-manifolds. Diff. Geom. Appl. 29 (2011), no. 3, 319-328.

[27] Roytenberg, D. and Weinstein, A.: Courant algebroids and strongly homotopy Lie algebras. Lett. Math. Phys., 46 (1998), no. 1, 81-93. 
[28] Roytenberg, D.: On the structure of graded symplectic supermanifolds and Courant algebroids. Quantization, Poisson Brackets and Beyond, 169-185, Contemp. Math., 315, Amer. Math. Soc., Providence, RI, 2002.

[29] Roytenberg, D.: Quasi-Lie bialgebroids and twisted Poisson manifold. Lett. Math. Phys. 61 (2002), no. 2, $123-137$.

[30] Roytenberg, D.: Courant algebroids, derived brackets and even symplectic supermanifolds. Ph. D thesis, UC Berkeley, 1999, arXiv:math.DG/9910078.

[31] Roytenberg, D.: On weak Lie 2-algebras. XXVI Workshop on Geometrical Methods in Physics. 180-198. AIP Conf. Proc., 956. Amer. Inst. Phys., Melville, NY, 2007.

[32] Schätz, F.: Coisotropic submanifolds and the BFV-complex. Ph. D thesis, University Zürich, 2009

[33] Ševera, P.: Some title containing the words "homotopy" and "symplectic", e.g. this one. Travaux mathématiques. Fasc. XVI, 121-137, Trav. Math., XVI, Univ. Luxemb., Luxembourg, 2005.

[34] Ševera, P. and Weinstein, A.: Poisson geometry with a 3-form background. Prog. Theor. Phys. Suppl., 144 (2001), 145-154.

[35] Sheng, Y. and Zhu, C.: Higher Extensions of Lie Algebroids. Comm. Contemp. Math. (2016), DOI: 10.1142/S0219199716500346.

[36] Sheng, Y. and Zhu, C.: Semidirect products of representations up to homotopy. Pacific J. Math., 249 (2011), no. 1, 211-236.

[37] Sheng, Y. and Zhu, C.: Integration of semidirect product Lie 2-algebras. Int. J. Geom. Methods Mod. Phys. 9 (2012), no. 5,1250043 .

[38] Stasheff, J.: Differential graded Lie algebras, quasi-Hopf algebras and higher homotopy algebras. Quantum groups (Leningrad, 1990), 120-137, Lecture Notes in Math., 1510, Springer, Berlin, 1992.

[39] Voronov, Th.: Graded manifolds and Drinfeld doubles for Lie bialgebroids. Contemp. Math. 315 (2002), $131-168$.

[40] Voronov, Th.: Higher derived brackets and homotopy algebras. J. Pure Appl. Algebra 202 (2005), no. 1-3, 133-153.

[41] Weinstein, A.: Omni-Lie algebras. Microlocal analysis of the Schrödinger equation and related topics (Japanese) (Kyoto, 1999). Sūrikaisekikenkyūsho Kūkyūroku 1176 (2000), 95-102.

[42] Xu, X.: Twisted Courant algebroids and coisotropic Cartan geometries. J. Geom. Phys. 82 (2014), 124-131. 